\title{
Tuned liquid dampers for multi-storey structure: numerical simulation using a partitioned FSI algorithm and experimental validation
}

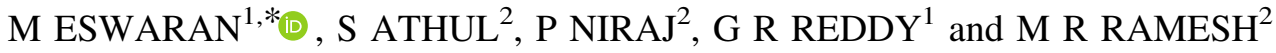 \\ ${ }^{1}$ Structural and Seismic Engineering Section, Reactor Safety Division, Bhabha Atomic Research Centre, \\ Mumbai 400085, India \\ ${ }^{2}$ National Institute of Technology Karnataka, Suratkal, Mangalore 575025, India \\ e-mail: eswarm21@gmail.com; eswaran@barc.gov.in
}

MS received 27 April 2016; revised 6 October 2016; accepted 20 October 2016

\begin{abstract}
Wind-induced and earthquake-induced vibrations of structures such as super-tall towers and bridges can be efficaciously controlled by tuned liquid dampers (TLDs). This work presents a numerical simulation procedure to study the performance of TLDs-structure system through sigma $(\sigma)$-transformation-based fluidstructure coupled solver. For this, a ' $\mathrm{C}$ '-based computational code has been developed. The structural equations, which are coupled with the fluid equations in order to achieve the transfer of sloshing forces to structure for damping, are solved by the fourth-order Runge-Kutta method, while the fluid equations are solved using finitedifference-based sigma-transformed algorithm. Different iterative and error schemes are used to optimize the code for larger convergence rate and higher accuracy. For validation, a few experiments are conducted with a three-storey structure using TLDs arrangement. The present numerical results of response of TLD-installed structures match well with the experimental results. The minimum displacement of structure is observed when the resonance condition of the coupled system is achieved through proper tuning of TLDs. Since real-time excitations are random in nature, the performance study of TLDs under random excitation has also been carried out in which the Bretschneider spectrum is used to generate the random input wave.
\end{abstract}

Keywords. Tuned liquid dampers; sloshing; $\sigma$-transformation; fluid-structure interaction; experiments.

\section{Introduction}

A partially filled water tank can be used as an effective damper for suppressing horizontal vibration of structures. This damper is called tuned liquid damper (TLD) because it utilizes the dynamic effect of liquid sloshing. A TLD is liquid confined in a container that uses the sloshing energy of the water to diminish the vibration of the system when the system is subjected to external excitation such as wind load and earthquake load. Several TLD systems can be kept in a structure to effectively control the structural oscillations with distributed sloshing frequencies over a range near the structural frequency and this is known as MTLD. MTLD has also been found to be very effective in revoking vibrations caused due to wind. However, in this work, several TLDs are used with same sloshing frequencies for mitigation of structural vibration. This damper is extremely practical and works on the principle of energy dissipation through liquid sloshing and wave breaking on the free surface. Fundamental mode frequency of liquid sloshing is

*For correspondence tuned to the natural frequency of the structure, and the damping ratio of the sloshing mode is set to an optimal value. As depicted by Tamura [1], the first TLD installation of an actual ground structure was performed successfully in Nagasaki Airport Tower (NAT), Nagasaki, Japan, in 1987. Now these dampers are used in many super-tall structures and bridges of developed countries, for example, in the John Hancock Tower in Boston, Citicorp Building in the New York and Ikuchi bridge and Sakitama bridge in Japan $[2,3]$.

When building a structure or component, it is important to calculate wind load to ensure that it can withstand high winds [4]. Mass and liquid dampers and base isolators are used to suppress the vibrations of the structures among the various alternatives. This paper will emphasize on one of these devices, TLD. A TLD consists of, partially filled liquid (usually water) tanks, which is typically situated on the top of a building. As the building moves in the extreme wind or earthquake attack, the fluid contained within the tank starts sloshing. Vibrational energy from the structure will be engrossed by the fluid, which transforms it into kinetic and potential energy of the sloshing fluid. Sloshing 
forces from the damper are used to mitigate structural responses. Since its inherent benefits include easy installation and handling, easy maintenance and lower cost, TLDs are gradually being used as vibration absorbers and have attracted increasing research interest in recent years [5]. Practical analysis of such dampers has been reported mainly for controlling structures due to wind loads $[1,6]$.

In this paper, a ' $\mathrm{C}$ '-based computational code is developed using finite-difference approach to capture the liquid free surface and liquid load estimation. Here the liquid free surface is captured using $\sigma$ transformation as it eliminates the requirement for free surface smoothing and re-meshing. The calculated liquid loads are effectively coupled with structural equations to estimate the displacement of a TLDcoupled structure.

\section{$1.1 \sigma$-Transformation technique}

Along with appropriate fluid equations, an additional algorithm is required to capture the moving free surface. For this, algorithms such as marker and cell (MAC) method, level set method (LSM), volume of fluid (VOF), etc. are commonly used during the simulation process [7-14]. The coordinate transformation technique can also be used to track the liquid free surface $[15,16]$. The particular type of coordinate transformation that features stretching in vertical direction is known as $\sigma$ transformation. This approach has earned reputation because of its simplicity, since all others requires a complex computer programming in order to treat the time-varying free surface boundary and update the computational mesh.

Initially, Phillips [17] proposed the $\sigma$-transformation technique for meteorological forecasting. Later, Blumberg and Mellor [18] applied this to oceanic and coastal flows. The $\sigma$ transformation was applied to nonlinear steep waves in fixed and base-excited tanks by Chern et al [19] and Turnbull et al [20]. Frandsen [21] studied steep free surface sloshing in fixed and base-excited rectangular tanks with a focus on moving liquid tank with horizontal and vertical excitations. Chen and Nokes [22], Dai and Xu [23], Eswaran et al [16], Wu et al [24] and Eswaran and Reddy [25] applied $\sigma$ transformation to appraise the sloshing effects on simple 2-D and 3-D tanks such as a horizontal cylindrical container and rectangular tank, etc. In this work, the $\sigma$ transformation is used to study the effectiveness of TLDs to track the free surface in fluid-structure coupled finite-difference solver.

\subsection{Problem definition and objective}

In order to find the structural displacement in TLD-coupled structure, many works approximate the behaviour of the liquid through the spring-mass model, as the actual liquid motion is complex and not amenable to exact mathematical modelling. This spring-mass model is available for only simple geometries like rectangular and cylindrical tanks and procedures can be found in ACI [26] and Housner [27]. This model does not consider the nonlinear motion of the wave. The response of a TLD, even for small amplitude motion, is highly nonlinear due to liquid sloshing. For accurately capturing the motion of sloshing water in a vibrating tank, nonlinear functions must be employed. Hence, a dynamic fluid-structure coupled analysis is necessary. Therefore, it is necessary to develop a fluid-structure partitioned code for the accurate predictions of the structural motion, which is coupled with TLDs. Here, the fluid and structure equations are solved separately and effectively coupled in each iteration to study the performance of TLDs. The objectives of the present work are as follows.

(i) To develop a computational fluid-structure coupled algorithm for the TLDs with a three-storey structure (as shown in figures 1 and 2) under base excitation. The code is used to analyse the effectiveness of the TLDs in reducing the structural oscillations under regular and random excitation through a fluidstructure coupled solver.

(ii) To optimize the code performance using various iterative schemes, error norms, stopping criteria for larger convergence rate and accurate solutions.

(iii) To validate the numerical solution with experimental results for various cases to increase the code credibility.

(iv) To study the floor-wise performance of TLDs.

In order to accomplish this, a modified $\sigma$-transformation technique is used to map the asymmetric physical domain onto a rectangle computational domain, such that the moving free surface in the physical plane becomes a fixed plane surface in the computational domain. A finite-

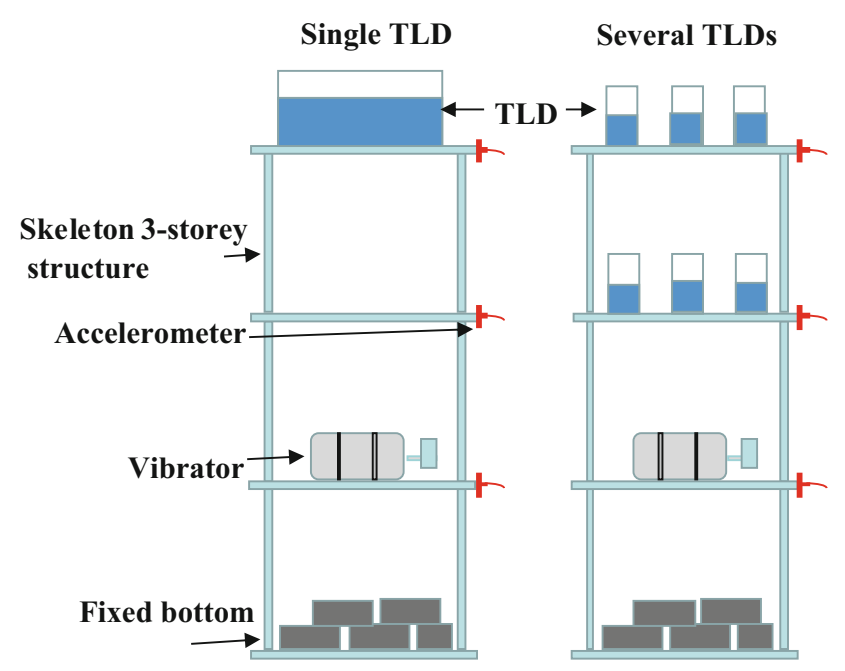

Figure 1. Simple TLD and TLDs arrangements in three-storey skeleton structure. 


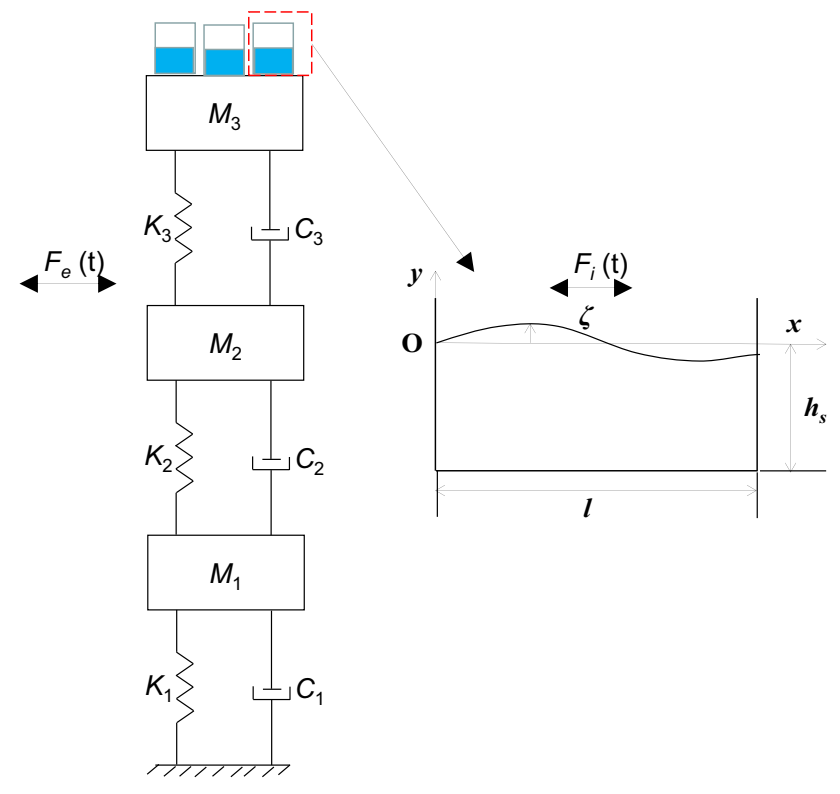

Figure 2. Schematic of a TLD-structure system with notations.

difference scheme is used to solve the discretized fluid governing equations in the transformed computational domain to obtain the free surface elevation time histories, phase plane diagram, spectrum analysis and free surface profiles in the physical domain. Structural equations are solved by the fourth-order Runge-Kutta method. Section 2 elaborates on the mathematical formulation of fluid, its mapping procedure and the structural formulations. In section 2.2, the finite-difference discretizations of the fluid governing equation and boundary conditions in the computational domain have been described. Experimental procedure is explained in section 3. After a performance analysis of various iteration schemes, error schemes and independence study, a detailed validation is performed with experiments. Experimental results are compared with numerical results qualitatively and quantitatively in section 4 . The concluding remarks are in section 5.

\section{Mathematical formulation}

\subsection{Fluid formulations}

Formulation for three-story structure-coupled TLDs is developed in this section. A rectangular Cartesian coordinate system is initially engaged with origin on the mean liquid free surface at the left wall of the container. Primarily, a nonlinear wave problem is considered, as depicted in figures 3 and 4 , where $\zeta$ is the free-surface elevation above still water level, $l$ is the length of the container and $h_{s}$ is the still water depth. Here, the fluid in the container is assumed to be inviscid and irrotational. Due to the

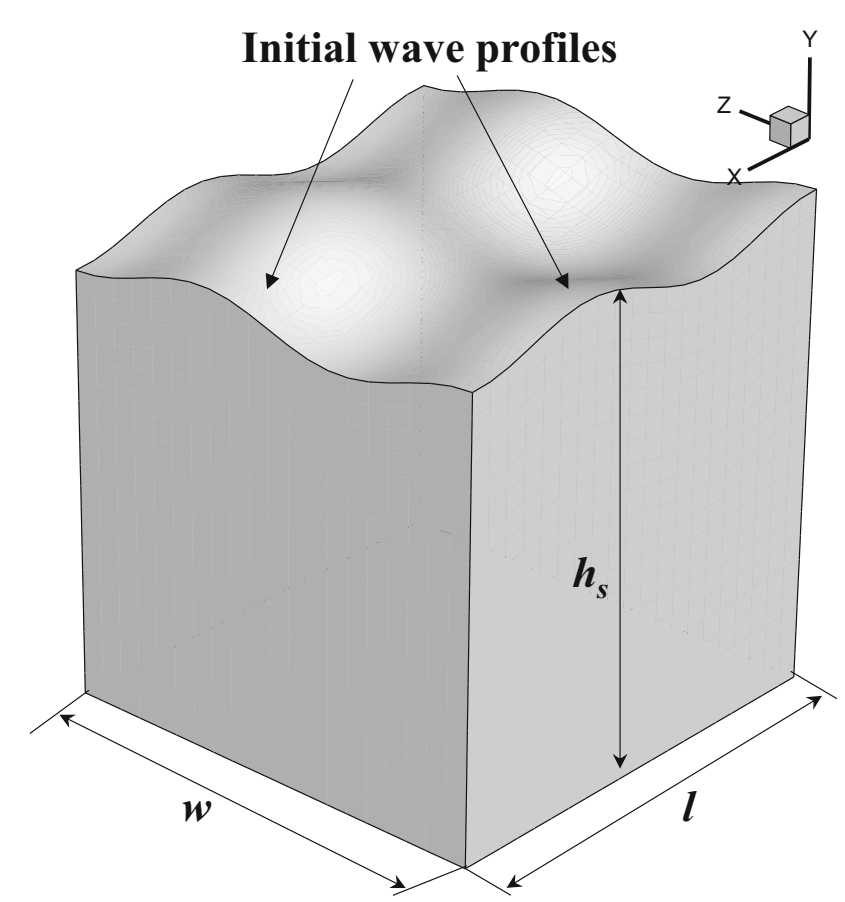

Figure 3. Physical domain with initial wave profiles 1 and 2 .

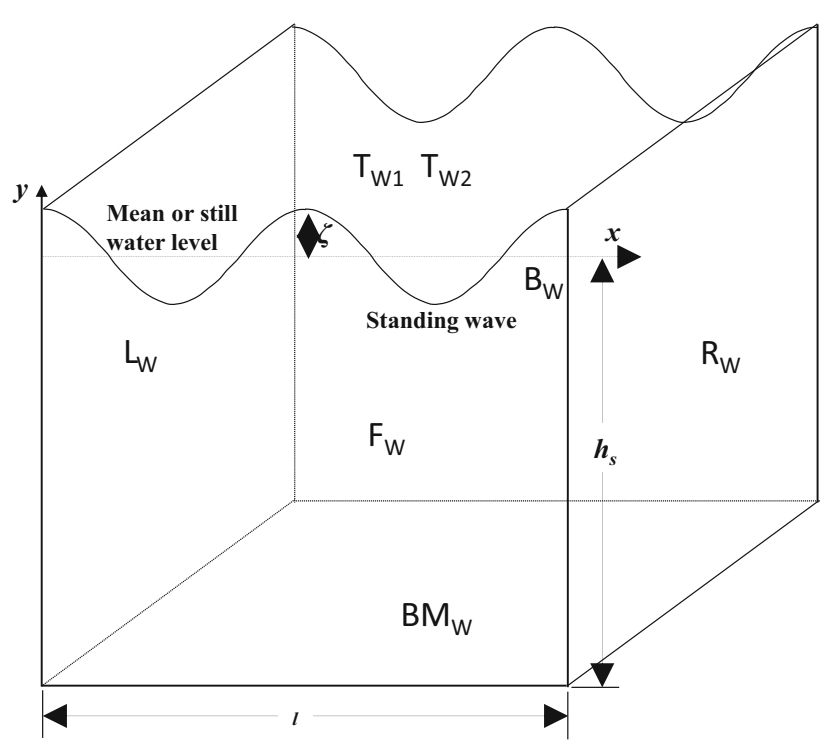

Figure 4. Sectional view of liquid tank with notations.

assumption that the fluid is governed by the potential flow theory, the velocity potential $\phi$ satisfies the Laplace equation. The velocity components normal to the fixed boundaries are zero by boundary condition. The left, right, front, back and bottom boundaries are indicated by $\mathrm{L}_{\mathrm{W}}, \mathrm{R}_{\mathrm{W}}, \mathrm{F}_{\mathrm{W}}$, $\mathrm{B}_{\mathrm{W}}$ and $\mathrm{BM}_{\mathrm{W}}$, respectively (figure 4). Two boundary conditions are to be applied at the free surface (i.e., the free surface occurs at the interface between two fluids). The first boundary condition is a kinematic condition that relates the 
motion of the free interface to the fluid velocities at the free surface (i.e., $\mathrm{T}_{\mathrm{W} 1}$ ), while the second one is a dynamic condition, which is concerned with the force balance at the free surface (i.e., $\mathrm{T}_{\mathrm{W}_{2}}$ ). Liquid velocity components normal to the walls and bottom of the container are zero. Zero pressure at the free surface of the fluid is also assumed in the analysis. In view of the afore-mentioned fact, kinematic and dynamic conditions must be satisfied on the free surface. The following quantities are introduced for generating dimensionless governing equations for the present study $[16,28]$ :

$$
\begin{gathered}
x^{\prime}=\frac{x}{l} ; \quad y^{\prime}=\frac{y}{l} ; \quad w^{\prime}=\frac{w}{l} ; \quad \zeta^{\prime}=\frac{\zeta}{A} \quad \ddot{X}_{t}^{\prime}=\frac{\ddot{X}_{t}}{g} ; \\
\ddot{Y}_{t}^{\prime}=\frac{\ddot{Y}_{t}}{g} ; \quad \ddot{Z}_{t}^{\prime}=\frac{\ddot{Z}_{t}}{g} ; \quad t^{\prime}=\sqrt{\frac{g}{l}} t ; \quad \phi^{\prime}=\frac{1}{A \sqrt{l} g} \phi
\end{gathered}
$$

where $g$ is the acceleration due to gravity; $l, w$ are, respectively, the length and width of the rectangular liquid damper. Here, $\ddot{X}_{t}, \ddot{Y}_{t}$ and $\ddot{Z}_{t}$ are the acceleration of the containers in the horizontal, vertical and lateral directions, respectively, which can be neglected from the free surface dynamic boundary condition for fixed container analysis. $A$ is the wave amplitude, and $t$ is the time.

The forcing characteristics amplitude is calculated as $A_{f}=F / A$, where $F$ is the total external force applied to the system. The $f_{f}$ is the force parameter $\left(=A_{f} / \rho g h^{2}\right)$ and it is a measure of nonlinearity. Hereafter, primes are omitted for simplification; the non-dimensional governing equation and boundary conditions can be written as follows:

$$
\begin{gathered}
\frac{\partial^{2} \phi}{\partial x^{2}}+\frac{\partial^{2} \phi}{\partial y^{2}}+\frac{\partial^{2} \phi}{\partial z^{2}}=0 \\
\mathrm{~L}_{\mathrm{W}} \text { and } \mathrm{R}_{\mathrm{W}}: \frac{\partial \phi}{\partial x}=0 \text { on } x=0, l \\
\mathrm{~F}_{\mathrm{W}} \text { and } \mathrm{B}_{\mathrm{W}}: \frac{\partial \phi}{\partial z}=0 \text { on } z=0, w \\
\mathrm{BM}_{\mathrm{W}}: \frac{\partial \phi}{\partial y}=0 \text { on } y=-h_{s} \\
\mathrm{~T}_{\mathrm{W} 1}: \frac{\partial \zeta}{\partial t}+f_{f} \frac{\partial \phi}{\partial x} \frac{\partial \zeta}{\partial x}+f_{f} \frac{\partial \phi}{\partial z} \frac{\partial \zeta}{\partial z}=\frac{\partial \phi}{\partial y} \text { on } y=\zeta \\
\mathrm{T}_{\mathrm{W} 2}: \frac{\partial \phi}{\partial t}+f_{f}\left(\frac{1}{2} \nabla \phi \nabla \phi\right)+\left(1+\ddot{Y}_{t}\right) \zeta+x \ddot{X}_{t}+z \ddot{Z}_{t} \\
=0 \text { on } y=\zeta
\end{gathered}
$$

Eqs. (2)-(7) form an initial boundary value problem, which is the Laplace equation with nonlinear boundary conditions imposed on the free surface. Here, the nonlinearity of the free surface is significant for two reasons: (i) the elevation of the moving free surface is not known a priori and (ii) the boundary conditions on the free surface (i.e., Eqs. (6) and (7)) contain second-order differential terms or products of unknown parameters.

2.1a Mapping procedure: Through proper coordinate transformations, the time-varying liquid free surface can be mapped onto a fixed plane surface by the $\sigma$ transformation, which prevents the need for free surface smoothing for the cases considered herein. In this paper, $\sigma$ transformation is applied in the horizontal direction, which stretches between the left and the right wall and in the vertical direction it stretches between the moving liquid free surface and the bottom of the liquid container, to convert the moving freesurface physical domain onto a fixed square computational domain. The following section discusses the transformation technique in a 3-D container in detail. In this formulation portion, the longitudinal and lateral excitation terms are removed from free surface boundary equations. During transformation, the corresponding governing equation and boundary conditions will change appropriately.

$2.1 \mathrm{~b}$ Coordinate transformation: This transformation adopted the $\sigma$-transformation technique to map the liquid domain onto a rectangle, such that the moving free surface in the physical plane (figures 4 and 5) becomes a fixed horizontal line in the $\sigma$-transformed domain (figure 6 ). The mapping function $\sigma(x, z, t)$ is defined as

$$
\sigma=\frac{y+h_{s}}{h}
$$

where

$$
h(x, z, t)=I_{b} \zeta(x, z, t)+h_{s} .
$$

Here, $\sigma(x, t)$ is the stretching factor, which varies from 0 to 1 . The value of $\sigma$ at the bottom of the container is 0 , while at the free surface it is 1 . The first-order derivatives of $\sigma$ can be calculated as follows:

$$
\frac{\partial \sigma}{\partial t}=\frac{\partial}{\partial t}\left(\frac{y+h_{s}}{h}\right)=-I_{b} \frac{\sigma}{h} \frac{\partial \zeta}{\partial t} \text { and } \frac{\partial \sigma}{\partial x}=-I_{b} \frac{\sigma}{h} \frac{\partial \zeta}{\partial x} .
$$

The potential function $\phi(x, y, z, t)$ in the physical domain is transformed to the potential function $\Phi(X, \sigma, Z, T)$ in the $\sigma$-transformed domain. The mapping relations for first transformation are as follows:

$$
X=2 x-1 ; Y=2 \sigma-1 ; Z=2 z-1 \text { and } T=t .
$$

Hence the transformed governing equation becomes

$$
\frac{\partial^{2} \Phi}{\partial X^{2}}+K_{1} \frac{\partial \Phi}{\partial Y}-K_{2} \frac{\partial^{2} \Phi}{\partial X \partial Y}-K_{3} \frac{\partial^{2} \Phi}{\partial Z \partial Y}+K_{4} \frac{\partial^{2} \Phi}{\partial Y^{2}}+\frac{\partial^{2} \Phi}{\partial Z^{2}}=0
$$

where 


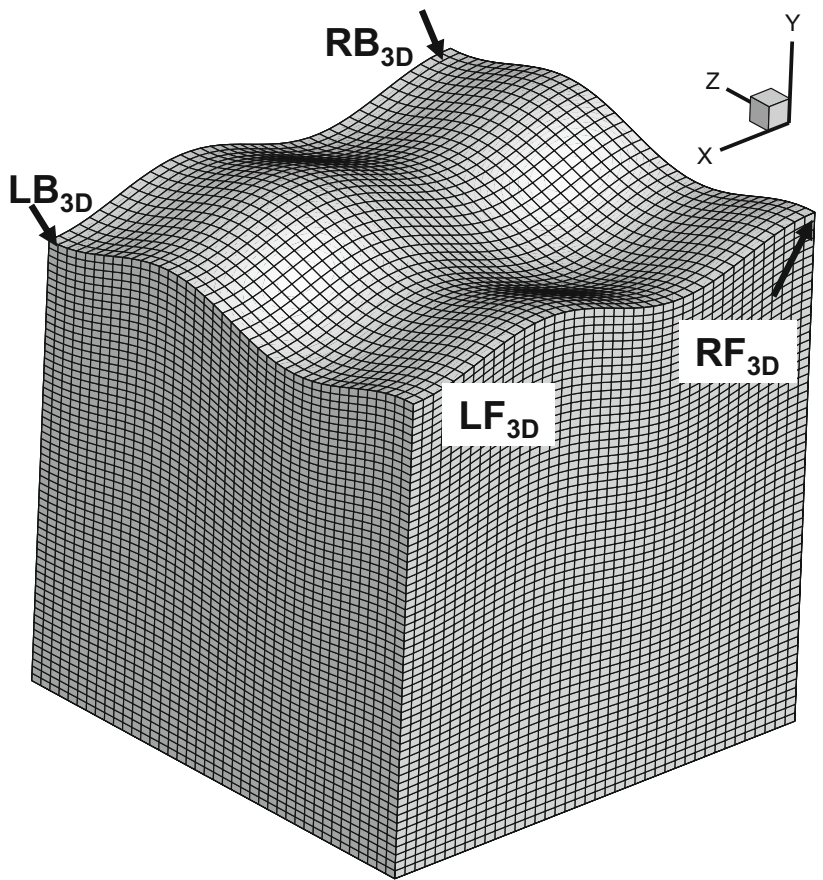

Figure 5. Physical domain with grid.

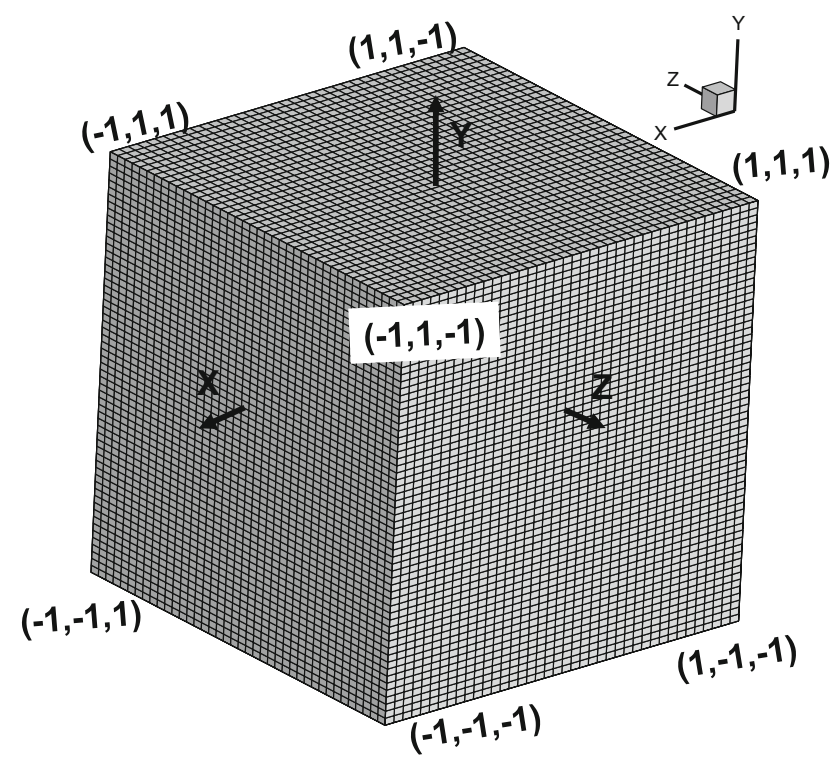

Figure 6. The 3-D intermediate domain after coordinate transformation.

$$
\begin{gathered}
K_{1}=\left[\frac{4 \sigma}{h^{2}}\left(\frac{\partial h}{\partial X}\right)^{2}-\frac{2 \sigma}{h} \frac{\partial^{2} h}{\partial X^{2}}+\frac{4 \sigma}{h^{2}}\left(\frac{\partial h}{\partial Z}\right)^{2}-\frac{2 \sigma}{h} \frac{\partial^{2} h}{\partial Z^{2}}\right] \\
K_{2}=\frac{2 \sigma}{h} \frac{\partial h}{\partial X} ; \quad K_{3}=\frac{2 \sigma}{h} \frac{\partial h}{\partial Z} ; \quad K_{4}=\left[\frac{1}{h^{2}}+K_{2}^{2}+K_{3}^{2}\right] .
\end{gathered}
$$

The boundary conditions are given as

$$
\begin{gathered}
\mathrm{L}_{\mathrm{W}} \text { and } \mathrm{R}_{\mathrm{W}}: \frac{\partial \Phi}{\partial X}-\frac{2 \sigma}{h} \frac{\partial h}{\partial X} \frac{\partial \Phi}{\partial Y}=0 \text { on } X=-1,+1 \\
\mathrm{~F}_{\mathrm{W}} \text { and } \mathrm{B}_{\mathrm{W}}: \frac{\partial \Phi}{\partial Z}-\frac{2 \sigma}{h} \frac{\partial h}{\partial Z} \frac{\partial \Phi}{\partial Y}=0 \text { on } \mathrm{Z}=-1,+1 \\
\mathrm{BM}_{\mathrm{W}}: \frac{\partial \Phi}{\partial Y}=0 \text { on } Y=-1 .
\end{gathered}
$$

On the free surface

$$
\begin{gathered}
\mathrm{T}_{\mathrm{W} 1}: \frac{\partial \zeta}{\partial T}=K_{5} \frac{\partial \Phi}{\partial Y}-4 I_{b} \frac{\partial \Phi}{\partial X} \frac{\partial \zeta}{\partial X}+-4 I_{b} \frac{\partial \Phi}{\partial Z} \frac{\partial \zeta}{\partial Z} \text { on } Y=+1 \\
\mathrm{~T}_{\mathrm{W} 2}: \frac{\partial \Phi}{\partial T}=+K_{6} \frac{\partial \Phi}{\partial Y}-\left(1+\ddot{Y}_{t}\right) \zeta+2 I_{b}\left[\left(\frac{\partial \Phi}{\partial X}-K_{7} \frac{\partial \Phi}{\partial Y}\right)^{2}\right. \\
\left.+\left(\frac{1}{h} \frac{\partial \Phi}{\partial Y}\right)^{2}+\left(\frac{\partial \Phi}{\partial Z}-K_{8} \frac{\partial \Phi}{\partial Y}\right)^{2}\right] \text { on } Y=+1
\end{gathered}
$$

where

$$
\begin{gathered}
K_{5}=\left[\frac{2}{h}+\frac{8 I_{b} \sigma}{h}\left(\frac{\partial \zeta}{\partial X}\right)^{2}+\frac{8 I_{b} \sigma}{h}\left(\frac{\partial \zeta}{\partial Z}\right)^{2}\right] ; \quad K_{6}=\frac{2 \sigma}{h} \frac{\partial h}{\partial T} \\
K_{7}=\frac{2 \sigma}{h} \frac{\partial h}{\partial X} ; \quad K_{8}=\frac{2 \sigma}{h} \frac{\partial h}{\partial Z} .
\end{gathered}
$$

2.1c Grid stretching near free surface: The mapping relations for second transformation are given as follows:

$$
\begin{aligned}
& X \leftrightarrow \xi, \quad \xi=X ; \quad Z \leftrightarrow \kappa, \quad \kappa=Z ; \quad T \leftrightarrow \tau, \tau=T ; \quad \text { and } \\
& Y \leftrightarrow \eta, \\
& \quad \eta=\alpha_{1}+\left(1-\alpha_{1}\right) \frac{\ln \left(\left(\alpha_{2}+\left[\frac{Y+1}{h_{c d}}\right]\right) /\left(\alpha_{2}-\left[\frac{Y+1}{h_{c d}}\right]\right)\right)}{\ln \left[\left(\alpha_{2}+1\right) /\left(\alpha_{2}-1\right)\right]} .
\end{aligned}
$$

The mapping is performed from the $\sigma$-transformed $\Phi(X, Y, Z, T)$ domain to the computational domain $\varphi(\xi, \eta, \kappa, \tau)$. The height of the liquid, $h(X, Z, T)$ surface, is transformed to the height of the liquid in the transformed computational domain $h_{*}(\xi, \kappa, \tau)$. The $h_{c d}$ is the height between two successive nodal points along the $Y$ axis of the grid after the first coordinate transformation. The derivatives of the function $\Phi(X, Y, Z, T)$ with respect to $X, Y, Z$ and $T$ are transformed into derivatives of $\varphi(\xi, \eta, \kappa, \tau)$ with respect to $\xi, \eta, \kappa$ and $\tau$. After the second coordinate transformation the governing equation in the fluid domain becomes 


$$
\begin{aligned}
& \frac{\partial^{2} \varphi}{\partial \xi^{2}}+C_{4} \frac{\partial \varphi}{\partial \eta}-C_{3} U_{1} \frac{\partial^{2} \varphi}{\partial \xi \partial \eta}-C_{3} U_{2} \frac{\partial^{2} \varphi}{\partial \kappa \partial \eta}+C_{5} \frac{\partial^{2} \varphi}{\partial \eta^{2}} \\
& +C_{6} \frac{\partial^{2} \varphi}{\partial \kappa^{2}}=0
\end{aligned}
$$

$$
\begin{gathered}
U_{9}=\frac{8 I_{b} \sigma^{*}}{h_{*}}\left(\left(\frac{\partial h_{*}}{\partial \xi}\right)^{2}+\left(\frac{\partial h_{*}}{\partial \kappa}\right)^{2}\right) ; \quad U_{7}=C_{3} \frac{2 \sigma^{*}}{h_{*}} \frac{\partial h_{*}}{\partial \xi} \\
U_{8}=C_{3} \frac{2 \sigma^{*}}{h_{*}} \frac{\partial h_{*}}{\partial \kappa} ; \quad U_{6}=C_{3} \frac{2 \sigma^{*}}{h_{*}} \frac{\partial h_{*}}{\partial \tau}
\end{gathered}
$$

where

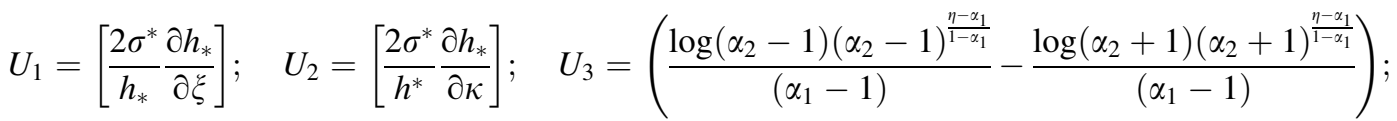

$$
\begin{aligned}
& U_{4}=\left(\frac{\log \left(\alpha_{2}-1\right)\left(\alpha_{2}-1\right)^{\frac{\eta-\alpha_{1}}{1-\alpha_{1}}}}{\left(\alpha_{1}-1\right)}+\frac{\log \left(\alpha_{2}+1\right)\left(\alpha_{2}+1\right)^{\frac{\eta-\alpha_{1}}{1-\alpha_{1}}}}{\left(\alpha_{1}-1\right)}\right) \\
& U_{5}=\left[\frac{1}{h_{*}^{2}}+\left(\frac{2 \sigma^{*}}{h_{*}} \frac{\partial h_{*}}{\partial \xi}\right)^{2}+\left(\frac{2 \sigma^{*}}{h_{*}} \frac{\partial h_{*}}{\partial \kappa}\right)^{2}\right] ; \quad C_{1}=\frac{\left(1-\alpha_{1}\right)}{\ln \left(\frac{\alpha_{2}+1}{\alpha_{2}-1}\right)} \times \frac{4 \alpha_{2} \times \sigma^{*} \times C_{2}}{\left(h_{c d} \times\left[\alpha_{2}^{2}-\sigma^{* 2}\right]^{2}\right)} ; \\
& C_{3}=\frac{\partial \eta}{\partial Y} ; \quad C_{5}=C_{3}^{2} \times\left[\frac{1}{h_{*}^{2}}+\left(\frac{2 \sigma^{*}}{h_{*}^{2}} \frac{\partial h_{*}}{\partial \xi}\right)^{2}+\left(\frac{2 \sigma^{*}}{h_{*}} \frac{\partial h_{*}}{\partial \kappa}\right)^{2}\right] ; \quad C_{7}=\frac{\partial \kappa}{\partial Z}
\end{aligned}
$$

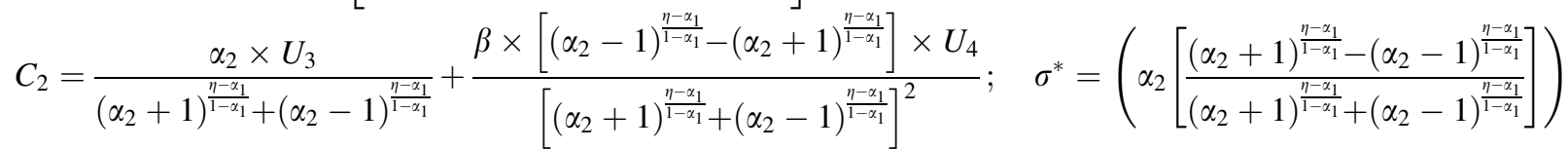

$$
\begin{aligned}
& C_{4}=\left(\frac{4 \sigma^{*}}{h_{*}^{2}}\left(\left[\frac{\partial h_{*}}{\partial \xi}\right]^{2}+\left[\frac{\partial h_{*}}{\partial \kappa}\right]^{2}\right)\right)-\frac{2 \sigma^{*}}{h_{*}}\left(\frac{\partial^{2} h_{*}}{\partial \xi^{2}}+\frac{\partial^{2} h_{*}}{\partial \kappa^{2}}\right)+C_{1} \times U_{5} ; \quad C_{6}=C_{7}^{2} \times\left[\frac{1}{h_{*}^{2}}+\left(\frac{2 \sigma^{*}}{h_{*}^{2}} \frac{\partial h_{*}}{\partial \xi}\right)^{2}+\left(\frac{2 \sigma^{*}}{h_{*}} \frac{\partial h_{*}}{\partial \eta}\right)^{2}\right] .
\end{aligned}
$$

Boundary conditions after the second transformation are given as follows:

$\mathrm{L}_{\mathrm{W}}$ and $\mathrm{R}_{\mathrm{W}}: \frac{\partial \varphi}{\partial \xi}-\frac{2 \sigma^{*}}{h_{*}} C_{3} \frac{\partial h_{*}}{\partial \xi} \frac{\partial \varphi}{\partial \eta}=0$ on $x=-1,1$

$\mathrm{F}_{\mathrm{W}}$ and $\mathrm{B}_{\mathrm{W}}: \frac{\partial \varphi}{\partial \kappa}-\frac{2 \sigma^{*}}{h_{*}} C_{3} \frac{\partial h_{*}}{\partial \kappa} \frac{\partial \varphi}{\partial \eta}=0$ on $\kappa=-1,1$

$$
\mathrm{BM}_{\mathrm{W}}: \frac{\partial \varphi}{\partial \eta}=0 \text { on } \eta=-1
$$

On the free surface,

$$
\begin{aligned}
\mathrm{T}_{\mathrm{W} 1}: \frac{\partial \zeta^{*}}{\partial \tau}= & -4 I_{b}\left(\frac{\partial \varphi}{\partial \xi} \frac{\partial \zeta}{\partial \xi}+\frac{\partial \varphi}{\partial \kappa} \frac{\partial \zeta}{\partial \kappa}\right) \\
& +C_{3}\left[\frac{2}{h_{*}}+U_{9}\right] \frac{\partial \varphi}{\partial \eta} \text { on } \eta \\
= & 1
\end{aligned}
$$

$$
\begin{aligned}
\mathrm{T}_{\mathrm{W} 2}: \frac{\partial \varphi}{\partial \tau}= & -2 I_{b}\left[\left(\frac{\partial \varphi}{\partial \xi}-U_{7} \frac{\partial \varphi}{\partial \eta}\right)^{2}+\left(\frac{\partial \varphi}{\partial \kappa}-U_{8} \frac{\partial \varphi}{\partial \eta}\right)^{2}\right]^{*} \\
& -2 I_{b}\left(\frac{C_{3}}{h_{*}} \frac{\partial \varphi}{\partial \eta}\right)^{2}+U_{6} \frac{\partial \varphi}{\partial \eta}-\left(1+Y_{\tau}^{*}\right) \zeta \text { on } \eta=1
\end{aligned}
$$

where
The stretched grid system will exactly match the timedependent free-surface wave profile due to the $\sigma$ transformation. Since the mean water level in the container remains constant, the sum of the wave height along the $x$ direction is kept at zero to maintain the volume conservation. Since sloshing is a highly nonlinear phenomenon, the amplitude of excitation, frequency of excitation and container dimensions play a vital role in determining the shape of the nonlinear free surface. An amplitude-frequency relationship for the fluid response in a two-dimensional rectangular container is presented by Faltinsen et al [29].

\subsection{Finite-difference discretization in the computational domain}

In this work, the finite-difference scheme is used for liquid sloshing simulation. The governing equation and boundary conditions in the computational domain (Eqs. (21)-(28)) are discretized using the finite-difference method. The Adams-Moulton scheme is utilized for the computation of nonlinear solutions. As the Adams-Moulton scheme requires results from previous time steps to calculate the current time step result, a semi-implicit scheme is used to generate the initial time steps. 
2.2a Semi-implicit scheme at top boundary (free surface): A semi-implicit scheme has been applied at the top boundary of the computational domain. As discussed, the top boundary consists of two conditions, viz., kinematic condition and dynamic condition. In computational domain, the kinematic condition (Eq. (26)) is discretized by a forward scheme at $\eta=1$ as follows:

$$
\left.\frac{\partial \zeta^{*}}{\partial \tau}\right|^{n}=\left.L_{4} C_{3} \frac{\partial \varphi}{\partial \eta}\right|^{n}-\left.\left.4 I_{b} \frac{\partial \zeta}{\partial \xi}\right|^{n} \frac{\partial \varphi}{\partial \xi}\right|^{n}
$$

where

$$
\left[\frac{\partial \zeta}{\partial T}\right]^{n}=\frac{\zeta^{n+1}-\zeta^{n}}{\Delta T}
$$

At $\sigma=1$, the dynamic condition (Eq. (27)) is discretized as follows:

$$
\begin{aligned}
\left.\frac{\partial \varphi}{\partial \tau}\right|^{n} & =\left.C_{3} L_{5} \frac{\partial \varphi}{\partial \eta}\right|^{n}-\left.\left(1+Y_{\tau}^{*}\right) \zeta^{*}\right|^{n+1} \\
& -2 E_{b}\left[\left(\left.\frac{\partial \varphi}{\partial \xi}\right|^{n}-\left.C_{3} L_{6} \frac{\partial \varphi}{\partial \eta}\right|^{n}\right)^{2}+\left(\left.\frac{C_{3}}{h_{*}^{2}} \frac{\partial \varphi}{\partial \eta}\right|^{n}\right)^{2}\right]
\end{aligned}
$$

where

$$
\left[\frac{\partial \varphi}{\partial T}\right]^{n}=\frac{\varphi^{n+1}-\varphi^{n}}{\Delta T} .
$$

The value of $\zeta^{n+1}$ is computed using Eq. (29) and is substituted in Eq. (30). Then, the new $\varphi^{n+1}$ is found at the top boundary of the computational domain using Eq. (32). The first- and second-order derivatives in these equations are discretized by means of a second-order central difference scheme within the liquid domain, and second-order forward and backward differences are employed at the boundaries.

2.2b Adams-Moulton scheme: Generally, Adams methods are known as explicit schemes because current and previous time step values are used to obtain the values for future time steps. However, the Adams-Moulton scheme is a set of multi-step methods, which are known as implicit methods. This method is initiated using either a set of known results or from the results of a Runge-Kutta method to start the initial value problem. It uses the explicit scheme to estimate the initial guess and uses the value to estimate the future time steps. Here, a four-point Adams-Moulton scheme is used as follows.

Predictor step: Adam-Bashforth four-point explicit scheme for the initial guess.

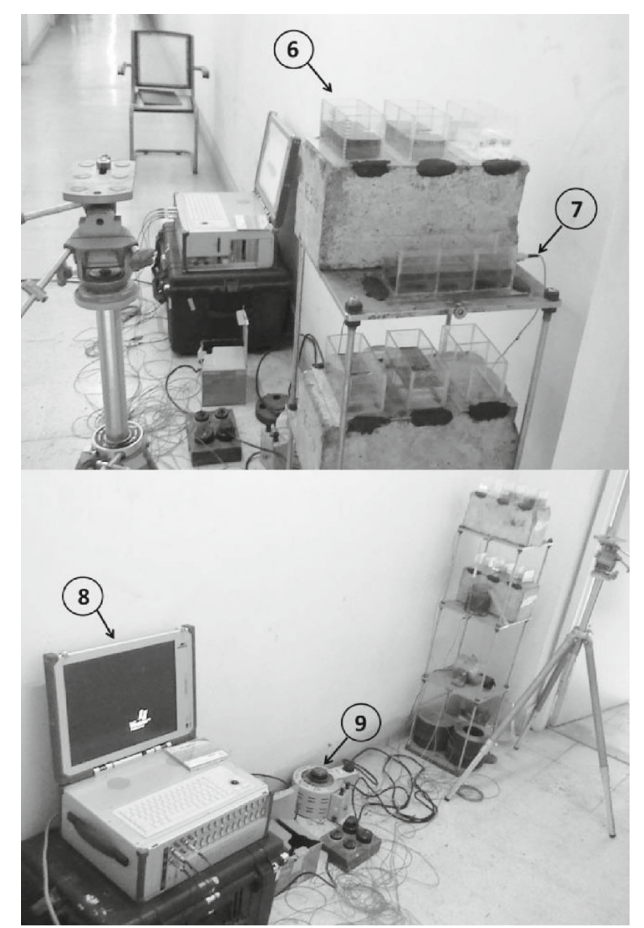

1. Camera setup to record slosh height; 2. Weight for fixed support; 3. Motor with eccentric weight for external vibration; 4. Extra weight; 5 . Skeletal structure; 6 . TLDs; 7. Acceleration probe; 8. Data acquisition system; 9. Auto-transformer for voltage variation.

Figure 7. Experimental set-up of three-storey structure with TLDs. 


$$
\left(\Delta \varphi^{n+1}\right)^{*}=\frac{\Delta t}{24}\left[55 \varphi^{n}-59 \varphi^{n-1}+37 \varphi^{n-2}-9 \varphi^{n-3}\right]
$$

and

$$
\left(\varphi^{n+1}\right)^{*}=\varphi^{n}+\left(\Delta \varphi^{n+1}\right)^{*}+O\left(\Delta t^{4}\right) .
$$

Corrector step: Use the Adam-Moulton three-point implicit scheme to take a second step.

$$
(\Delta \varphi)^{\mathrm{n}+1}=\frac{\Delta h}{24}\left[9\left(\varphi^{n+1}\right)^{*}+19 \varphi^{n}-5 \varphi^{n-1}+\varphi^{n-2}\right]
$$

and $\left(\varphi^{n+1}\right)=\varphi^{n}+(\Delta \varphi)^{n+1}+O\left(\Delta t^{4}\right)$.

2.2c Discretization: The governing equation (Eq. (21)) is discretized by the standard fourth-order central difference approximation and is given by

$$
\begin{aligned}
\delta_{\xi}^{2}= & \frac{-\varphi_{i-2, j}+16 \varphi_{i-1, j}-30 \varphi_{i, j}+16 \varphi_{i+1, j}-\varphi_{i+2, j}}{12\left(\Delta \xi^{2}\right)} \\
+ & O\left(\xi^{4}\right) \\
\delta_{\xi} \delta_{\eta}= & \frac{1}{2 \Delta \xi}\left(\frac{\varphi_{i+1, j+1}-\varphi_{i+1, j-1}}{2(\Delta \eta)}-\frac{\varphi_{i-1, j+1}-\varphi_{i-1, j-1}}{2(\Delta \eta)}\right) \\
& +O\left(\xi^{2}, \eta^{2}\right)
\end{aligned}
$$

where $\varphi(i, j)$ denotes $\varphi\left(\xi_{i}, \eta_{j}\right) ; \delta_{\xi}, \delta_{\xi}^{2}$ and $\delta_{\eta}, \delta_{\eta}^{2}$ are the firstand second-order central difference operators along $\xi$ and $\eta$ directions, respectively, and $\delta_{\xi} \delta_{\eta}$ is the mixed second-order central difference operator.

\subsection{Structural formulation}

2.3a Formulations for three-storey structure: In this study, a three-storey skeleton structure has been taken as shown in figures 1 and 7 . The equations for individual floor have to be formulated in such a way that the updated displacement, velocity and acceleration values of the previous floor should be incorporated in solving the equation of the current floor. The structure equations floor-wise are calculated as follows:

$$
\text { for 1st floor, } \begin{aligned}
M_{1} \ddot{d}_{1}= & -k d_{1}+k\left(d_{2}-d_{1}\right)-c \dot{d}_{1}+c\left(\dot{d}_{2}\right. \\
& \left.-\dot{d}_{1}\right)+F_{\text {exc }}+F_{i 1}
\end{aligned}
$$

for 2nd floor, $M_{2} \ddot{d}_{2}=-k\left(d_{2}-d_{1}\right)+k\left(d_{3}-d_{2}\right)-c\left(\dot{d}_{2}\right.$

$$
\left.-\dot{d}_{1}\right)+c\left(\dot{d}_{3}-\dot{d}_{2}\right)+F_{i 2}
$$

for 3rd floor, $M_{3} \ddot{d}_{3}=-k\left(d_{3}-d_{2}\right)-c\left(\dot{d}_{3}-\dot{d}_{2}\right)+F_{i 3}$.

The terms $d_{1}, \dot{d}_{1}$ and $\ddot{d}_{1}$ represent the displacement, velocity and acceleration of floor 1 , respectively. The total

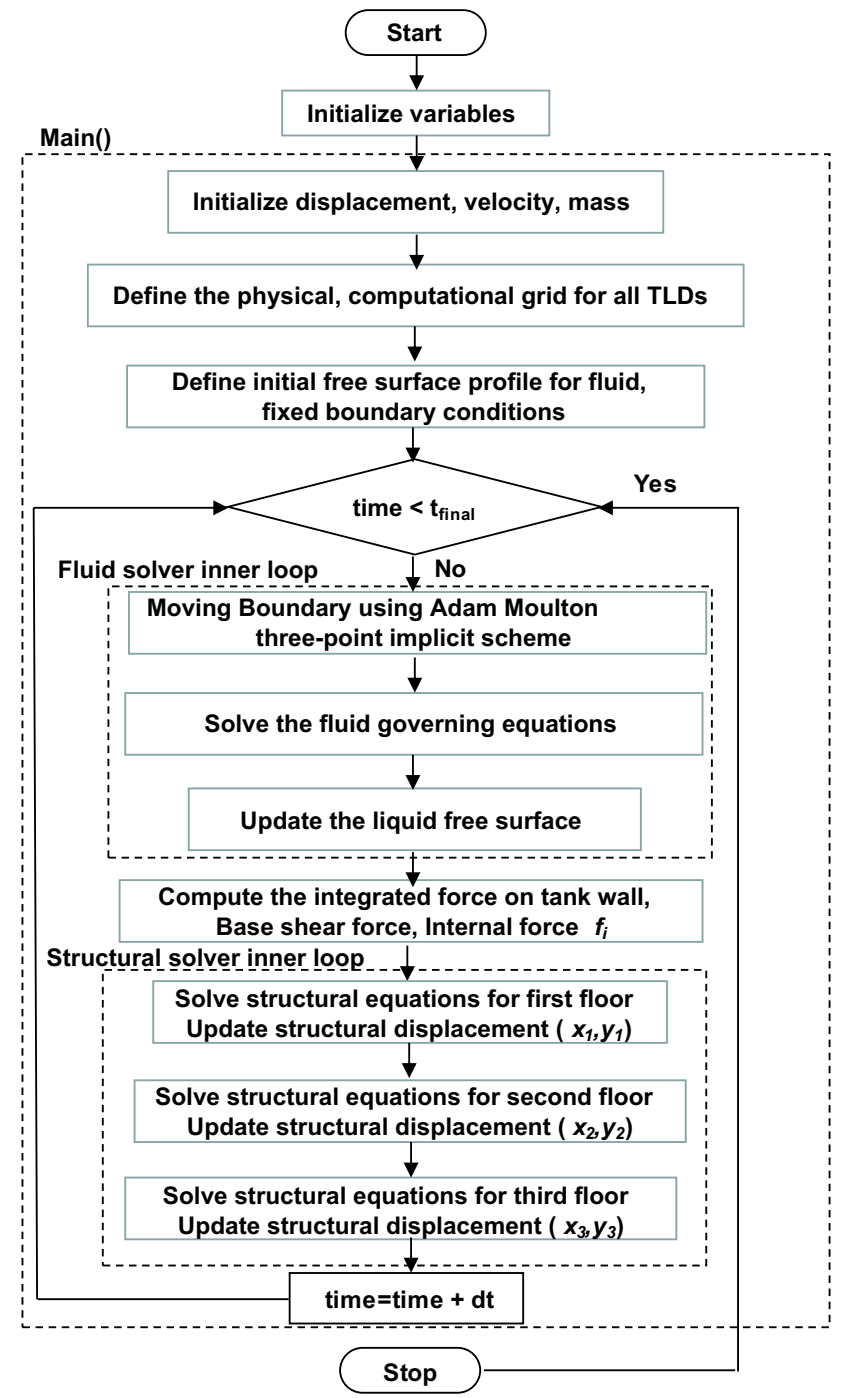

Figure 8. Flowchart of the present numerical algorithm.

fluid mass of TLDs is calculated, by virtue of which the structure mass is obtained; mass ratio is maintained at $1 \%$. The total structure mass is divided to the floor masses in such a way that the masses $M_{1}, M_{2}$ and $M_{3}$ carry equal values initially. The total TLDs mass is then added to the respective floor carrying the TLDs. The effective floor stiffness $k$ is evaluated from the structural stiffness so that the frequency of the structure is made equivalent to that of the TLDs. The floor-wise damping $c$ is calculated from the respective mass and stiffness with a minimum damping ratio of $0.13 \%$. The external excitation force is taken as $F_{\text {exc }}=A \cos (\omega t)$, which is included in the equation of first floor to serve as the base excitation. Here $A$ and $\omega$ are, respectively, the excitation amplitude and frequency where $t$ is the time. The internal force due to liquid sloshing of TLDs system for first, second and third floors is denoted as $F_{i 1}, F_{i 2}$ and $F_{i 3}$, respectively. The internal force developed 
due to the action of liquid sloshing at 3rd floor is given as follows:

$$
\text { internal force }\left(F_{i 3}\right) \text { at } 3 \text { rd floor }=B_{S F 3}+P_{F 3}
$$

where $B_{S F 3}$ and $P_{F 3}$ are the total liquid shear force from 3rd floor dampers and total force due to pressure on tank walls from 3rd floor dampers, respectively. Base shear of a damper $\left(B_{S F}\right)$ in physical domain is calculated as follows:

$$
B_{S F}=\frac{1}{2} \rho g\left(\bar{\zeta}(0, z, t)^{2}-\bar{\zeta}(b, z, t)^{2}\right)
$$

Liquid slosh force for a damper $\left(P_{F}\right)$ can be obtained by integrating the pressure values over the right and left tank walls:

$$
F_{i}(t)=\int_{-h_{s}}^{\zeta} p(x, y, t) d y ; \text { over } x=0 \text { and } b
$$

where pressure $(p)$ is obtained from the unsteady Bernoulli equation in the physical domain:

$$
\frac{p}{\rho}=-\frac{\partial \phi}{\partial t}-g y-x X_{t}^{\prime \prime}-\frac{1}{2}(\nabla \phi)^{2}
$$

over the left and right tank walls. The initial conditions are such that the structure is assumed to be at rest and the water surface is still and undisturbed:

$$
\begin{aligned}
\bar{\phi}(t & =0)=0 ; \bar{\zeta}(t=0)=0 ; X_{t}^{\prime \prime}(0)=F_{e}(0) / m ; X_{t}(0) \\
& =0 \text { and } X_{t}^{\prime}(0)=0 .
\end{aligned}
$$

Many schemes are available to solve the structural nonlinear time integration equations. In this work, structural equations are solved by the fourth-order RungeKutta method, since the present system has moderate vibrations.

\subsection{Fluid-structure coupled solver}

Structural equations are coupled with fluid equations in order to achieve the transfer of sloshing forces to structure for damping. A numerical algorithm for the three-storey structure is developed and a flowchart of the algorithm is illustrated in figure 8. In order to incorporate the damping action of TLDs into the structure, the resisting internal forces due to sloshing are calculated floor-wise by adopting the acceleration developed in the previous or bottom floor, which is included in the equation of the respective floor. In case of random excitation, the present external force equation is replaced by the force developed due to the acceleration generated by the Bretschneider spectrum, which serves to be the source for random input waves.

\subsection{Convergence criteria used for the iterative solver}

Each and every numerical algorithm necessarily solves a linear equation $A \phi=B$ in the computational domain at every time step. To solve this equation, various methods are available such as the simply implicit procedure (SIP) [30-32], bi-conjugate gradient stabilized method (BiCGSTAB) [33], preconditioned bi-conjugate gradient stabilized method (PBiCGSTAB) [32], etc. De [32] found that the rate of convergence improves significantly from the SIP to the PBiCGSTAB technique while BiCGSTAB lies between them with non-monotonic convergence. Figure 9 shows the number of iterations required for Gauss-Seidel successive over-relaxation (GS-SOR), SIP, BiCGSTAB and PreBiCGSTAB schemes. The PreBiCGSTAB iterative method has been chosen for this work to find the solution to the velocity potential $\phi$ at every time step since these methods have the advantages of lesser discretization error and better speed of computation as compared with other direct methods as shown in figure 10. The volume error is computed for $n=1 ; \Omega_{x}=1.253 ; f_{f}=0.0033$ and $K_{x}=0.015$ over non-dimensional time, where $\Omega_{x}$ and $K_{x}$ are liquid frequency ratio (= excitation frequency to natural frequency) and wave number in $x$ direction, respectively.

While using an iterative solver it is very important to stop the iterations at the right time, i.e., when the iteration error has decreased by an acceptable amount. The volume errors for all the cases studied are found to be within the range of $\left[-10^{-8}, 10^{-8}\right]$ as shown in figure 11 .

Various iterative solver stopping criteria are used for optimization of the present code using the simple tank problem as shown in figure 11 . It can be noted that the difference norm falls well below $0.1 \times 10^{-8}$ by 50

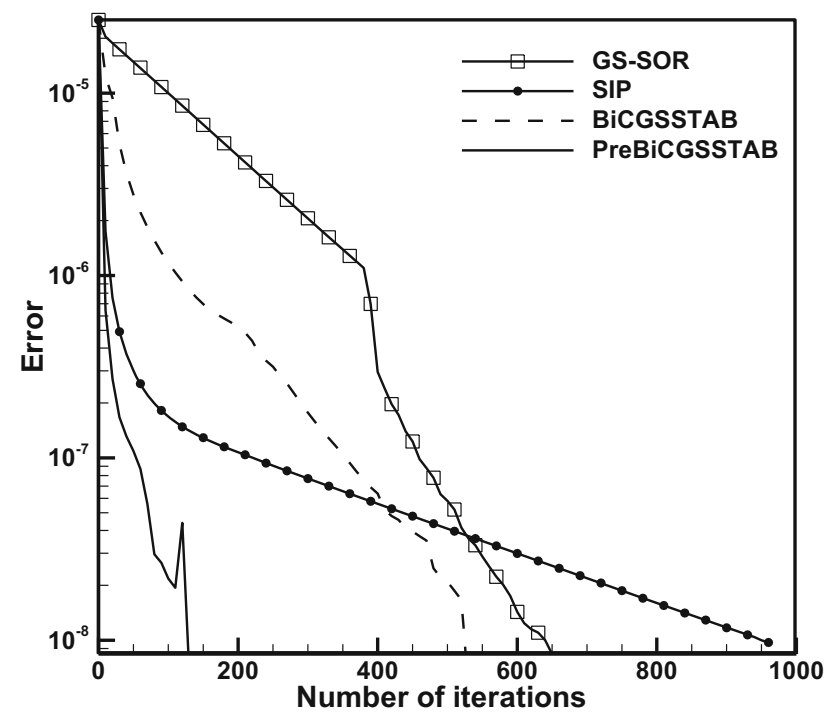

Figure 9. Comparison of iteration schemes. 


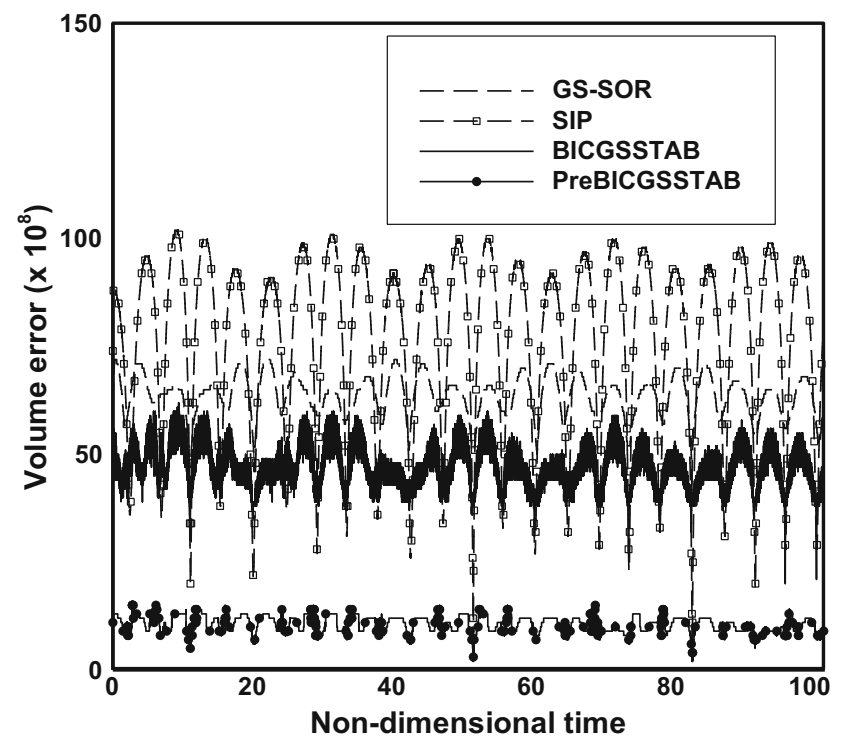

Figure 10. Volume error (for $n=1 ; \Omega_{v}=1.253 ; K_{v}=0.4$; $E_{b}=0.0033$ and $\left.K_{x}=0.015\right)$ versus non-dimensional time $(\omega t)$.

iterations, whereas the exact iteration error is still above $0.3 \times 10^{-5}$ and hence this stopping criterion is not suited for this case. Taking into account the residual norm, it is clear that it has quite a higher value than the exact iteration error for all iterations. The estimated iteration error is almost the same as the exact iteration error in this case (except for the initial iterations, where it shows slight oscillations). The residual stopping criterion ensures that the exact iteration error has fallen well below the tolerance limit, but it results in higher computational time for the solver without achieving higher accuracy than the estimated iteration error stopping criterion. Comparisons made for later time steps also showed a similar behaviour of the norms of the stopping criteria with iterations. Hence, the error estimation stopping criterion with the PreBiCGSTAB iterative scheme is the most suited for the current problem. As shown in figure 12, grid independence study is performed and $61 \times 61 \times 61$ grid size is taken for the present study.

\section{Experimental procedure}

An experimental study is conducted to evaluate the performance of TLDs and their effectiveness in reducing the structural response. TLD tanks made of acrylic sheets of thickness $4 \mathrm{~mm}$ are fabricated. The tanks are made leak proof by applying a combination of resin powder and chloroform at the edges. The length, width and height of the tanks are taken as $50 \mathrm{~mm} \times 50 \mathrm{~mm} \times 60 \mathrm{~mm}$. A threestorey skeletal structure is taken for study as shown in figure 7. The height of each floor is $300 \mathrm{~mm}$, which

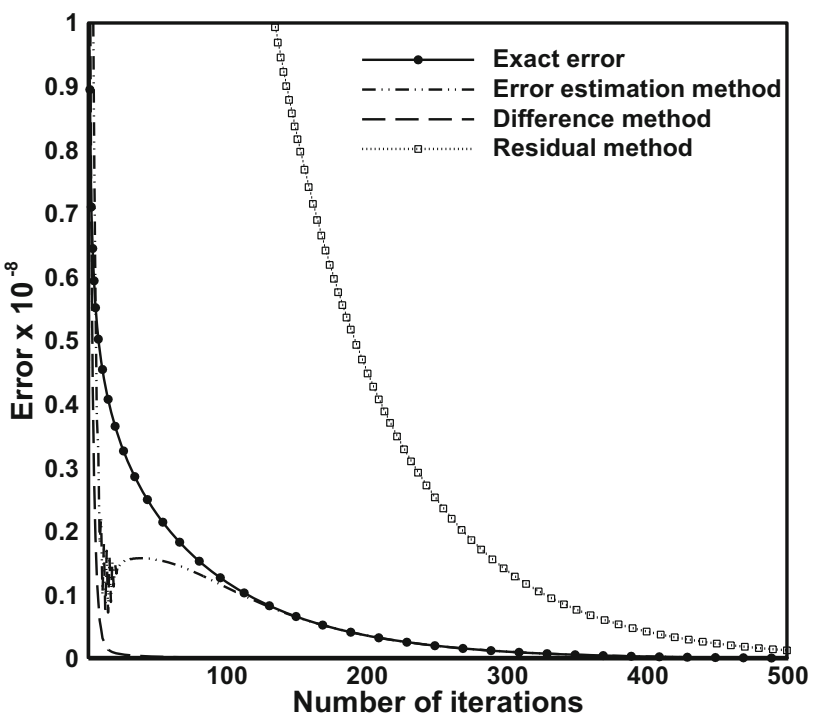

Figure 11. Comparison of error norms.

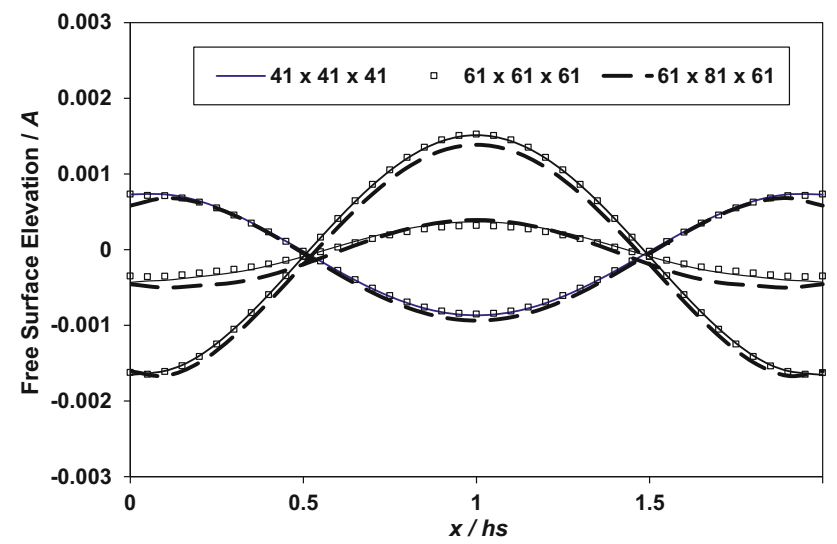

Figure 12. Grid independence study.

occupies a flat plate of dimensions $300 \mathrm{~mm} \times 300 \mathrm{~mm}$ and a thickness of $10 \mathrm{~mm}$. The ground floor movement is constrained by the addition of heavy weights. An exciter (motor connected with an eccentric weight and dimmerstat) is attached to the first floor, which acts as the source of external vibration. The frequency of excitation can be altered by the use of dimmerstat, by virtue of which the voltage is regulated to obtain the desired frequency of excitation. The limitation of this exciter is its ability to excite only in one direction. The top two floors are utilized for placing liquid dampers as shown in figure 7. Acceleration probes are attached to the floors, which are connected to the data acquisition system for recording the data. A camera is fixed on a stand in order to capture the slosh height. 


\section{Results and discussion}

The effect of the liquid sloshing on the structural motion is established by the fluid-to-structure mass ratio $M_{r}$ and tuning ratio relative to the structure $\Omega_{t}=\omega_{n} / \omega_{0}$ where $\omega_{n}$ and $\omega_{0}$ are the sloshing frequency of mode $n$ and first mode structural frequency, respectively. The free-surface behaviour is investigated by varying the external forcing amplitude through the forcing parameter $\left(f_{f}=A_{f} / \rho g h^{2}\right)$.

\subsection{Code validation with experiments}

To prove the credibility of the present numerical code, results need to be compared with the experimental results. Experiments are conducted as explained in section 3. The natural frequency of TLDs is tuned with a structural system by adjusting the water fill level and is kept on the threestorey structure as shown in figure 7 . This case involves qualitative comparison between numerical and experimental results by showing the free surface profile at different time instants with five TLDs at the top floor as shown in figure 13. Snapshots of the experimental and numerical free surface profile are shown and it is observed that the numerical results are in good agreement with the experiment.

\subsection{Effect of forcing frequency ratio $(\beta)$}

$\beta\left(=\omega / \omega_{0}\right)$ is defined as the ratio of excitation frequency to first mode structural frequency. This case study features the effect of frequency ratio at $\Omega_{t}=1, M_{r}=0.01$ and $f_{f}=0.0001$. Figure 14 shows the non-dimensional displacement variation over non-dimensional time with and without five-TLDs at the top floor. It is seen that the structural displacement significantly reduces with the application of the TLD when it is tuned to the structural natural frequency. Figure 15 illustrates the fast Fourier transformation (FFT) plot of displacement with the TLD
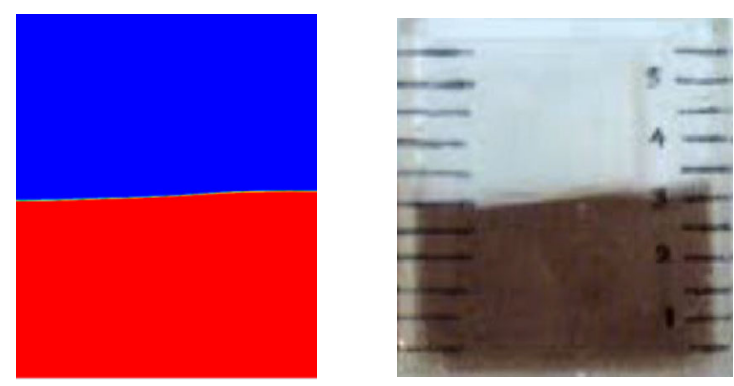

(a) $0.5 \mathrm{~s}$
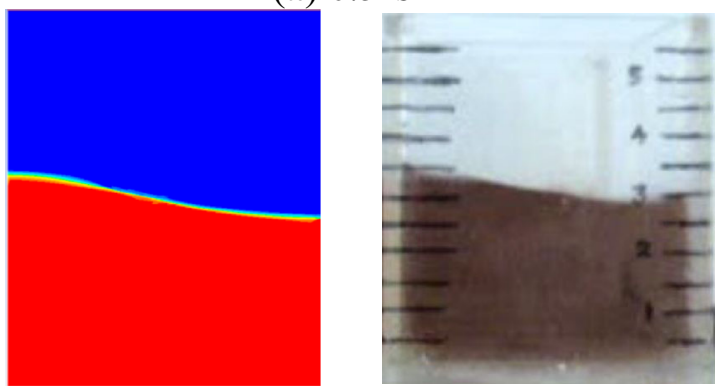

(c) $1.8 \mathrm{~s}$
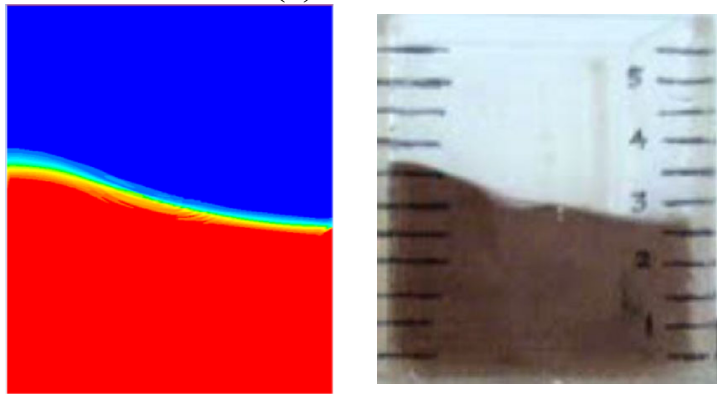

(e) $3.9 \mathrm{~s}$
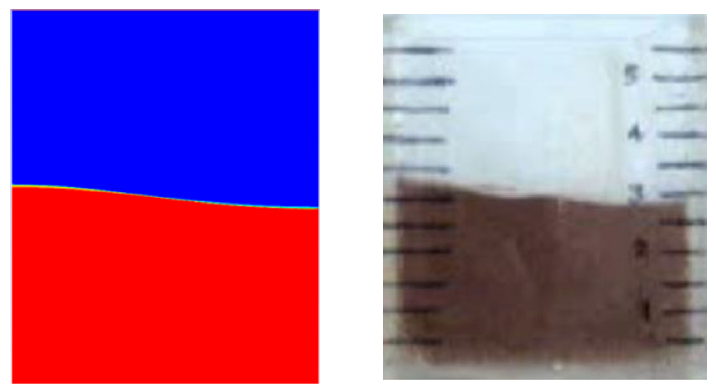

(b) $1 \mathrm{~s}$
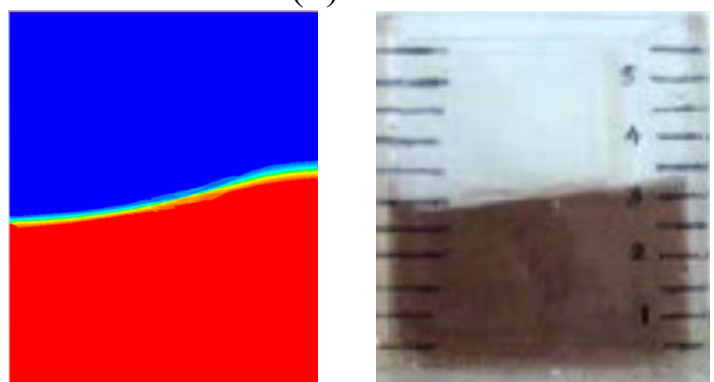

(d) $3.25 \mathrm{~s}$
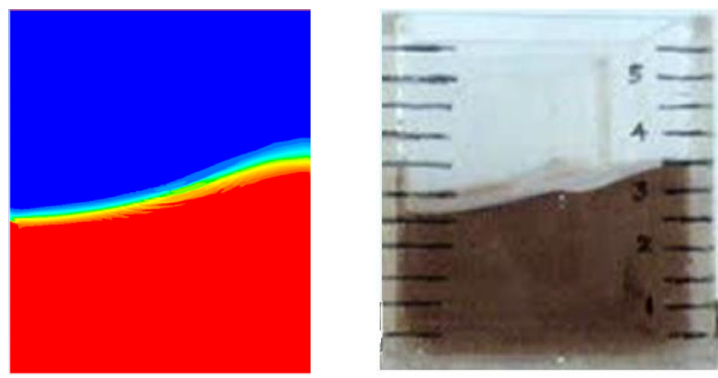

(f) $4.3 \mathrm{~s}$

Figure 13. Qualitative comparison of free surface profile for experimental and numerical results at different time instants. 


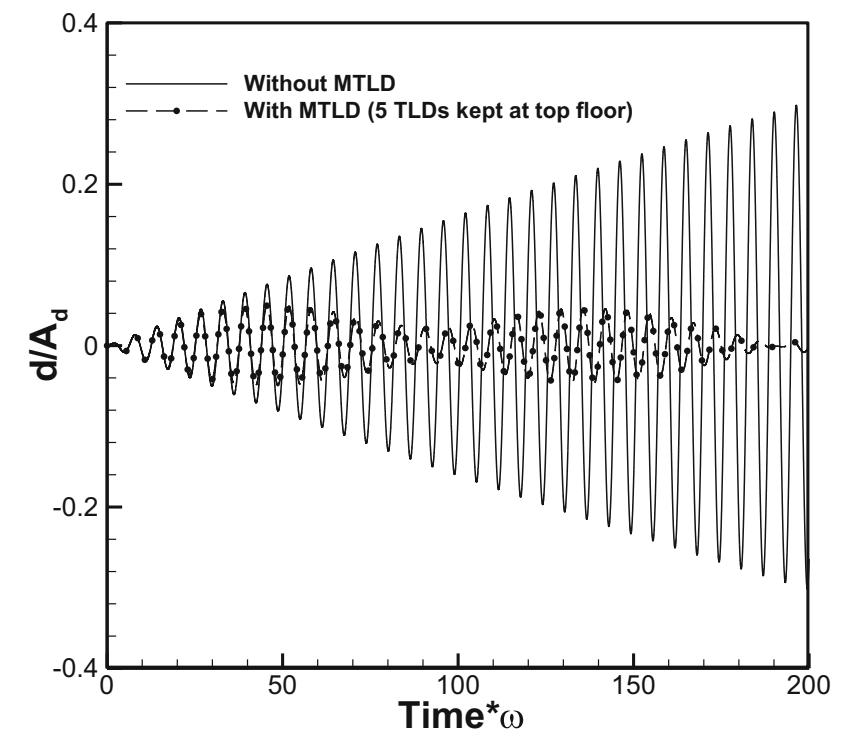

Figure 14. Displacement plot with and without TLD.

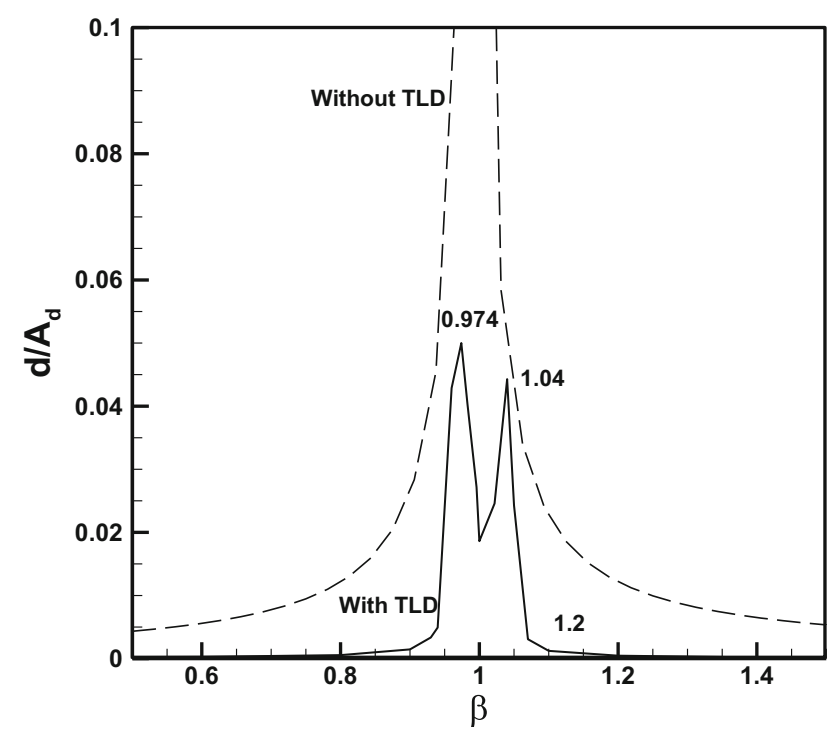

Figure 15. FFT plot of displacement curve with TLD.

case in which two dominant frequencies are obtained. Frandsen [28] discussed about the existence of two distinct frequencies for an effective tank-structure coupled system and also described the two values as eigen-frequencies of the system, obtained by solving the equations of motion through an analytical approach. Here, it is observed that the maximum response is found at $\beta=0.974$ and 1.04 as presented in figure 15. There will be a rise in amplitude of the corresponding sloshing mode due to the structural oscillations, when one of the sloshing frequencies is close to the structural natural frequency. This increase in sloshing mode begins to interact with the structure, thereby resulting in a change in the natural frequency of the coupled system.

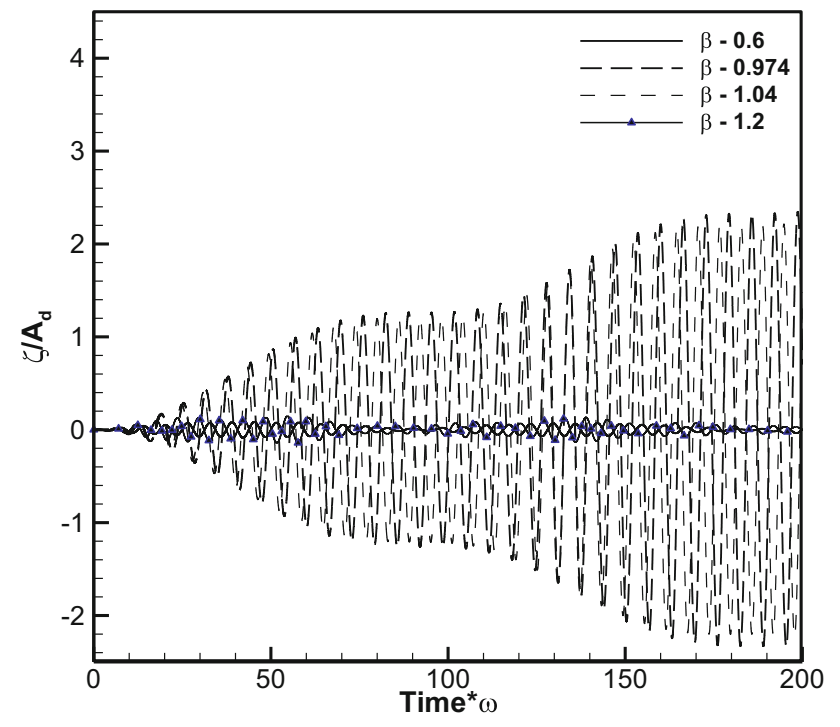

Figure 16. Displacement plot at various $\beta$ values.

To lend credibility to the numerical code, the results are further compared with the experimental results quantitatively for different $\beta$. Quantitative validation with the numerical code for selected cases has been performed and the structural displacement is illustrated in figures 16, 17, $18,19,20$ and 21. For this purpose, the required parameters are taken as $h=1 \mathrm{~m}, \quad b / h=2, \quad M_{r}=1 \%, \Omega_{t}=1$, $f_{f}=1 \mathrm{E}-4, \xi=0.0013, M_{t}=202, k=291.013, c=0.63$ and nine TLDs kept at the top floor. Figure 16 shows the plot of non-dimensional displacement against non-dimensional time for different frequency ratios. For the lower frequency ratio, the numerical results exhibit an excellent match with the experiments as shown in figure 17 , whereas figure 18 shows the numerical and experimental structural displacement at $\beta=1.2$.

Due to violent motion of the structure, small deviation is found at $\beta=0.974$ (figure 19). The violent motion is caused because of the TLD frequency matching with a structural frequency, which leads the displacement sensor to over-prediction. The eccentric arrangement used creates a few additional frequencies in the experimental data. During the violent motion of a structure the difference becomes more due to the above issues. Numerical dissipation can be controlled by higher-order upwind schemes. However, dominant frequencies are the same in both cases. The repeatable patterns in the phase plane diagram in figure 20 show the nonlinear cyclic behaviour of the displacement curve. The effectiveness of TLDs in a single floor is shown in table 1 . The nine MLTDs are placed at the top floor and reduction of structural displacement is noted for various $M_{r}$ and $\beta$. The maximum reduction is found at $M_{r}=1.4 \%$ and $\beta=0.974$. As stated before, the TLD performance is more for $M_{r}$ between $1 \%$ and $5 \%$. 


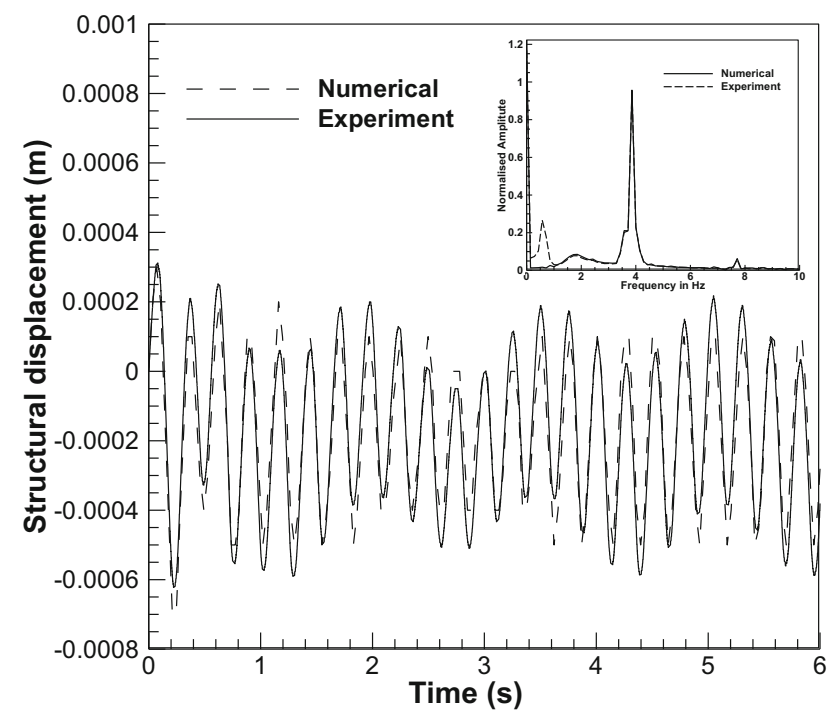

Figure 17. Experimetal and numerical comparison at $\beta=0.6$.

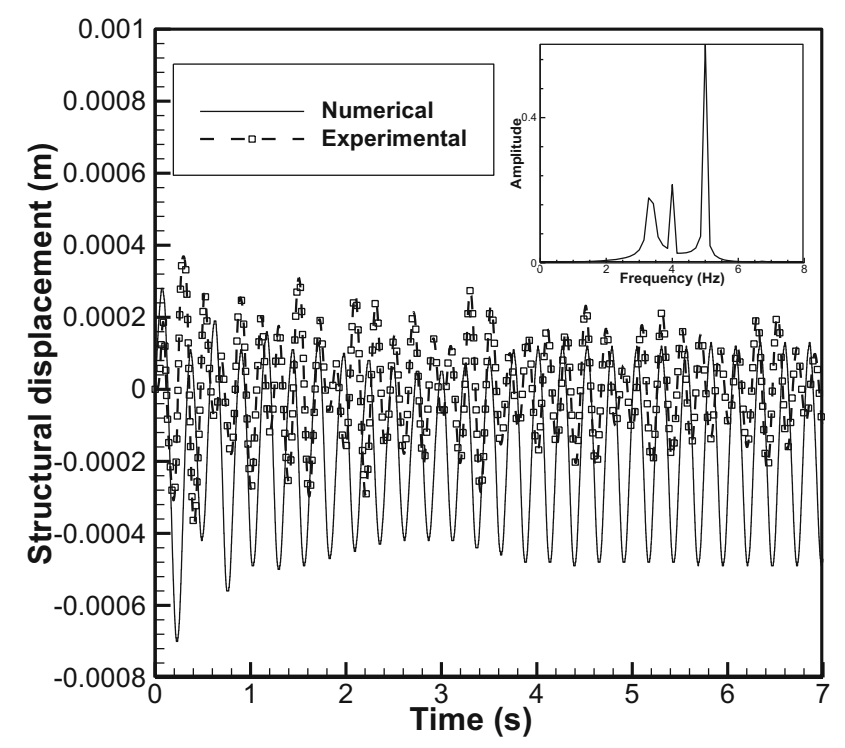

Figure 18. Experimetal and numerical comparison at $\beta=1.2$.

\subsection{Effect of TLDs in floor-wise numerical investigations}

The effect of introducing TLDs on structure floors is discussed in this section. The numerical predictions are put forward for different TLDs system configurations. Case studies of without TLD, three TLDs on the top floor and single TLD on each floor are conducted as shown in figure $21 \mathrm{a}, \mathrm{b}$ and $\mathrm{c}$, respectively. The results of the aforementioned cases under normal excitation and random excitation are illustrated in figures 22 and 23, respectively. In most practical situations, the excitation or the time variation of the system parameters is random in nature.

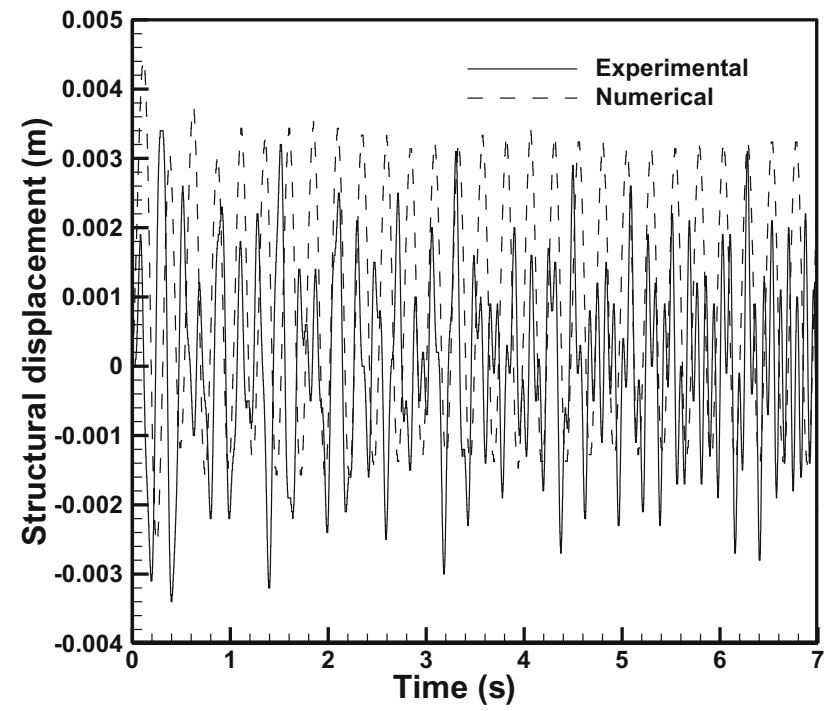

Figure 19. Experimetal and numerical comparison at $\beta=0.974$.

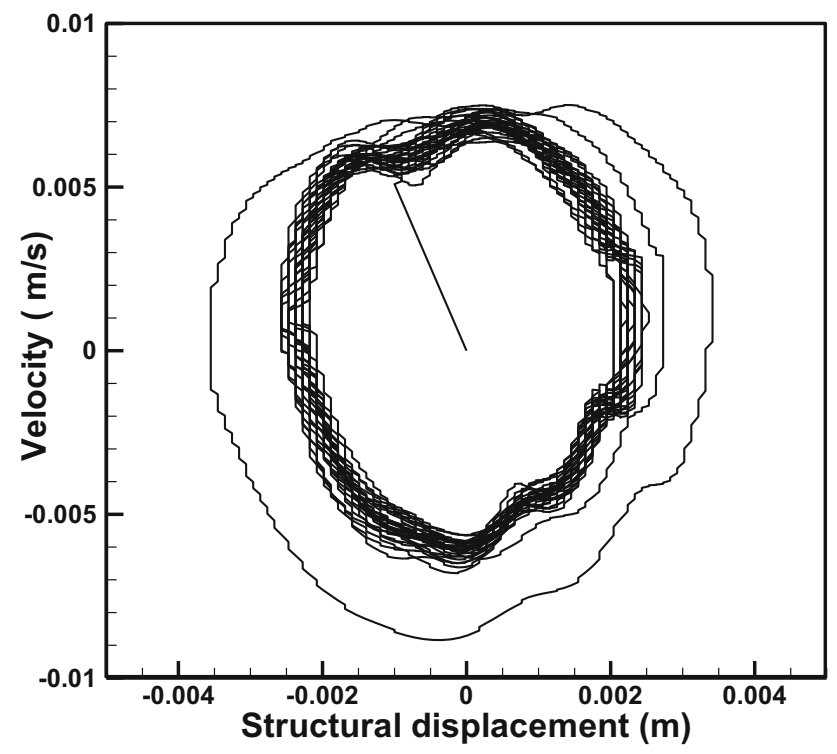

Figure 20. Numerical phase plane diagram $\mathrm{n}$ at $\beta=0.974$.

Hence, random motion has also been taken into consideration in this section. Linear superposition of a number of monochromatic waves can generate a random input wave. Vibration control dampers are usually designed based on random acceleration input such as real earthquake time history [34]. In this work, the Bretschneider spectrum has been selected to serve as the input excitation spectrum to generate the random input wave. A floating random number generating algorithm is used for the selection of linear angular frequencies and phase angle within the prescribed range. More details on the generation of random waves can be found in Sriram et al [35] and Eswaran et al [16]. 


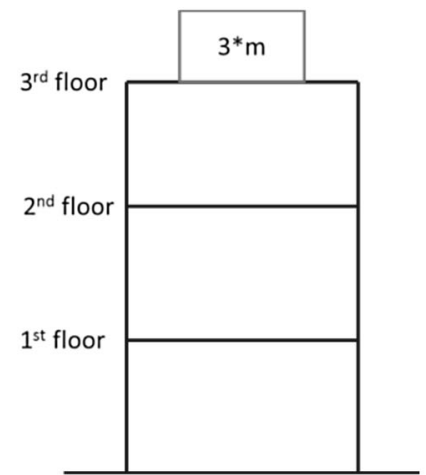

(a) Without TLD

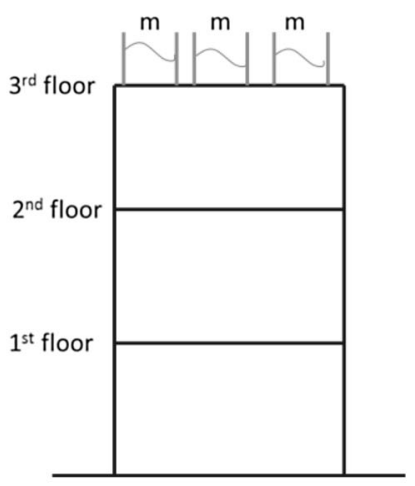

(b) 3 TLDs on top floor

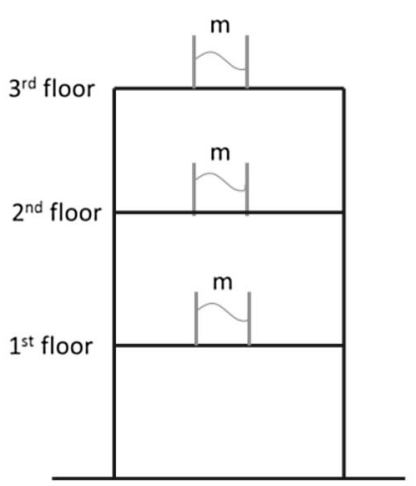

(c) Single TLD on every floor

Figure 21. Numerical case studies on multiple TLDs.

Table 1. Effectiveness of multiple TLDs in a single floor.

\begin{tabular}{|c|c|c|c|c|c|c|}
\hline \multirow[b]{2}{*}{ Method } & \multirow[b]{2}{*}{$\beta$} & \multirow[b]{2}{*}{ No. of TLDs } & \multirow[b]{2}{*}{ Mass ratio $M_{r}(\%)$} & \multicolumn{2}{|c|}{ Max. displacement in $m$} & \multirow[b]{2}{*}{ Reduction $(\%$} \\
\hline & & & & Without TLD & With TLD & \\
\hline Experiment & 0.6 & 9 & 0.56 & $4.5 \mathrm{E}-4$ & $2.5 \mathrm{E}-4$ & 44.44 \\
\hline Numerical & 0.6 & 9 & 0.56 & $4.5 \mathrm{E}-4$ & $2.5 \mathrm{E}-4$ & 44.44 \\
\hline Experiment & 0.974 & 9 & 0.56 & $2.1 \mathrm{E}-3$ & $3.3 \mathrm{E}-4$ & 84.2 \\
\hline Numerical & 0.974 & 9 & 0.56 & $2.2 \mathrm{E}-3$ & $3.2 \mathrm{E}-4$ & 85.45 \\
\hline Experiment & 0.974 & 9 & 1.4 & $5.3 \mathrm{E}-3$ & $3.5 \mathrm{E}-4$ & 93.40 \\
\hline Numerical & 0.974 & 9 & 1.4 & $5.3 \mathrm{E}-3$ & $3.7 \mathrm{E}-4$ & 93.02 \\
\hline
\end{tabular}

Displacement from top floor of the structure and $f_{T L D}=3.86, \mathrm{M}_{\mathrm{f}}=0.56 \mathrm{~kg}$.

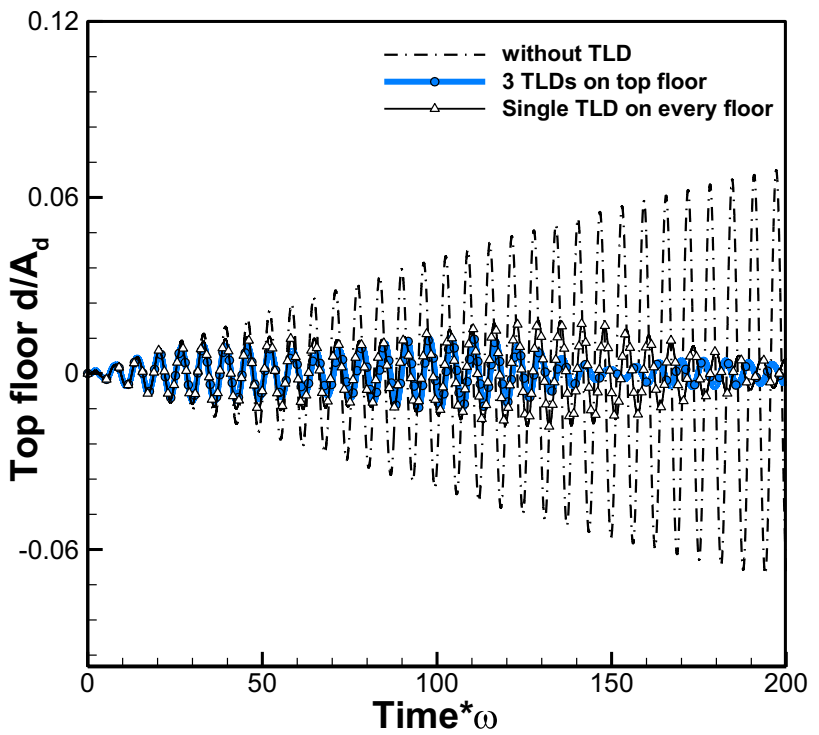

Figure 22. Non-dimensional displacement plot under normal excitation.

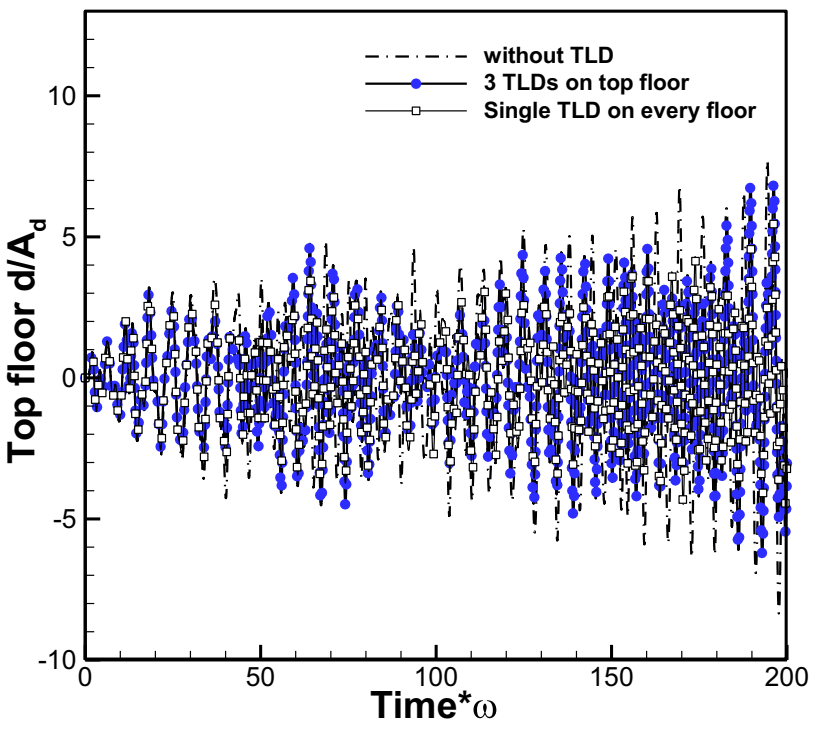

Figure 23. Non-dimensional displacement plot under random excitation. 
From figures 22 and 23, it can be observed that there is minimum structural displacement when the TLDs are placed on the top floor of the structure when compared with that arranged in individual floors. It can also be stated that the multiple TLDs arranged on a single floor provide more resisting sloshing forces required for damping the structural vibration. In the random case, the TLDs on top floor provided more damping initially but as time proceeds, both the cases show similar effects in mitigating structural response.

\section{Summary}

The international guidelines for liquid tank designs are based on the spring-mass model system. Since they are based on linear theory and also provide static liquid load, the estimation of a liquid load leads to imprecision. For accurate prediction, a dynamical fluid-structure coupled analysis is necessary. Therefore, it is also essential to develop a fluidstructure coupled code for the accurate predictions of the structural motion, which is coupled with TLDs.

In this work, the fluid and structure equations are solved separately and coupled effectively in each iteration to study the performance of TLDs. Structural equations are coupled with fluid equations in order to achieve the transfer of sloshing forces into the structure for damping using a partitioned FSI solver. Structural equations are solved by the fourth-order Runge-Kutta method while fluid equations are solved using a finite-difference-based sigma-transformed algorithm. Different iterative schemes and error schemes are used to optimize the code for larger convergence rate with higher accuracy. The error estimation stopping criterion with PreBiCGSTAB iterative scheme suits well for the current problem. Grid independence study has also been performed. To lend credibility to the numerical code, the computed results are compared with experimental data. For this, a detailed experiment on TLDs is conducted. The numerical results match well with the experimental results. The present numerical approach is easy to implement, computationally accurate and efficient. The cases presented herein eliminate the requirement for free surface smoothing and re-meshing. This model offers a simple way of simulating steep non-breaking waves.

It is clear from the above study that as the mass ratio is increased, the TLDs can effectively damp the structural vibration. In this case, the mass ratio is increased by increasing the number of TLDs actively participating in mitigating the structural vibration. Although the arrangement of TLDs in single floor seems to provide greater damping than that of dividing the same number of TLDs among the top two floors, the latter configuration proved effective in mitigating structural response in both of the perpendicular directions.

The numerical model is simple, computationally inexpensive and accurate and there is no necessity for free

surface smoothing. The present potential flow model may be readily extended to the prediction of magnetorheological (MR) fluid sloshing effects.

\section{Acknowledgement}

The authors wish to express their very sincere thanks to referees for their valuable comments and suggestions.

\section{List of symbols}

$A_{i}$ $A_{d}$ $A_{F}$ Amp $\alpha_{1}, \alpha_{2}$ $b$ $d_{i}, \dot{d}_{i}, \ddot{d}_{i}$ $h$ $h_{s}$ $H_{S}$ $N_{W}$ $\rho$ $g$ $\frac{g}{\zeta}$ $\bar{m}, \bar{c}, \bar{k}$

$D$

$\tau$

$\bar{\phi}$

$\bar{F}_{e}(t)$

$\bar{F}_{i}(t)$

$n$

$p$

$\omega_{n}$

$\omega_{1}$

$\omega_{0}$

$\omega$

$\omega_{i}$

$\omega_{p}$

$k_{n}$

$M_{r}$

$M_{t}$

$\xi$

$f_{f}$

$\Omega_{t}$

$\Omega_{x}$

$\beta$

$\phi(x, y, z, t)$ wave amplitude of $i$ th linear wave non-dimensional constant forcing characteristics amplitude external force characteristic amplitude grid stretching factors length of tank structural displacement, velocity and acceleration, respectively, of floor $i$ instant water height from tank bottom still water depth significant wave height number of linear monochromatic waves density of water acceleration due to gravity non-dimensional free surface elevation non-dimensional structure mass, damping and stiffness, respectively non-dimensional structural displacement non-dimensional time non-dimensional velocity potential function non-dimensional external force of excitation non-dimensional internal force of sloshing mode number of oscillation pressure the $n$th mode sloshing angular frequency first mode sloshing angular frequency structural natural frequency excitation angular frequency frequency of $i$ th linear wave peak frequency of wave spectrum wave number of $n$th mode mass ratio non-dimensional total mass damping ratio forcing factor tuning ratio liquid frequency ratio in $x$ direction forcing frequency ratio velocity potential function in physical domain $(x, y, z, t)$ 


$\begin{array}{ll}\Phi(X, Y, Z, T) & \begin{array}{l}\text { velocity potential function in } \\ \text { computational domain }(X, Y, Z, T) \\ m_{1}, m_{2}, n_{1}, n_{2}\end{array} \\ \begin{array}{l}\text { coordinate points on computational } \\ \text { domain axes } \\ S_{\zeta}\end{array} & \begin{array}{l}\text { Bretschneider spectrum wave energy } \\ \text { phase angle of } i \text { th linear wave }\end{array} \\ \psi_{i} & \text { base shear force } \\ \text { BSF } & \text { damping } \\ c & \text { computational fluid dynamics } \\ \text { CFD } & \text { external force } \\ F_{\text {ext }} & \text { fast Fourier transform } \\ \text { FFT } & \text { stiffness } \\ k & \text { mass of the floor } \\ M & \text { force due to pressure on tank walls } \\ \text { PF } & \text { time (s) } \\ t & \text { tuned liquid column damper } \\ \text { TLCD } & \text { tuned liquid damper } \\ \text { TLD } & \text { tuned sloshing damper } \\ \text { TSD } & \text { structural floor displacement } \\ x & \end{array}$

\section{References}

[1] Tamura Y 1995 Effectiveness of tuned liquid dampers under wind excitations. Eng. Struct. 17(9): 609-621

[2] Modi V J and Munshi S R 1998 An efficient liquid sloshing damper for vibration control. J. Fluid Struct. 12(8): 1055-1071

[3] Kaneko S and Ishikawa M 1999 Modeling of tuned liquid damper with submerged nets. J. Pressure Vessel Technol. 121(3): 334-343

[4] Eswaran M, Verma R K and Reddy G R 2016 Wind-induced loads and integrity assessment of hyperboloid reflector of solar power plants. Alexandria Eng. J. 55(2): 837-850

[5] Ruiz R O, Diego L G and Taflanidis A A 2016 Modeling and experimental validation of a new type of tuned liquid damper. Acta Mech. 85: 206-218

[6] Modi V J and Akinturk A 2002 An efficient liquid sloshing damper for control of wind induced instabilities. J. Wind Eng. Ind. Aerodyn. 90 (19): 1055-1071

[7] Jeon S H, Seo M W, Cho Y U, Park W G and Jeong W B 2013 Sloshing characteristics of an annular cylindrical tuned liquid damper for spar-type floating offshore wind turbine. Struct. Eng. Mech.: Int. J. 47(3): 331-343

[8] Eswaran M, Goyal P, Reddy G R, Singh R K and Vaze K K 2013 Fluid-structure interaction analysis of sloshing in an annular-sectored water pool subject to surge motion. Ocean Syst. Eng. 3(3): 1-21

[9] Eswaran M, Reddy G R and Singh R K 2015 Effect of higher modes and multi-directional seismic excitations on power plant liquid storage pools. Earthq. Struct. 8(3): 777-795

[10] Popov G, Sankar S, Sankar T S and Vatistas G H 1993 Dynamics of liquid sloshing in horizontal cylindrical road containers. Proc. Inst. Mech. Eng. Part C: J. Mech. Eng. Sci. 207(C6): 399-406

[11] Akyildiz H and Unal N 2006 Sloshing in a three-dimensional rectangular tank: numerical simulation and experimental validation. Ocean Eng. 33: 2135-2149
[12] Cho J R, Lee H W and Ha S Y 2005 Finite element analysis of resonant sloshing response in 2D baffled tank. J. Sound Vib. 288: 829-845

[13] Arafa M 2007 Finite element analysis of sloshing in rectangular liquid-filled tanks. J. Vib. Control 13: 883-903

[14] Sharma A 2015 Level set method for computational multifluid dynamics: a review on developments, applications and analysis. Sadhana 40(3): 627-652

[15] Hirt C W and Nichols B D 1981 Volume of fluid (VOF) method for the dynamics of free boundaries. J. Comput. Phys. 39: 201-205

[16] Eswaran M, Virk A S and Saha U K 2013 Numerical simulation of 3-D sloshing waves in a regularly and randomly excited container in vertical direction. J. Mar. Sci. Appl. 12(3): 298-314

[17] Phillips N A 1957 A coordinate system having some special advantages for numerical forecasting. J. Atmos. Sci. 14: 184-185

[18] Blumberg A F and Mellor G L 1978 A coastal ocean numerical model. In: Sundermann H J and Holz K P (Eds.) Proceedings of an international symposium on mathematical modeling of estuarine physics, Hamburg, Berlin: SpringerVerlag, pp. 203-219

[19] Chern M J, Borthwick A G L and Taylor R E 1999 A pseudospectral $\sigma$-transformation model of 2-D nonlinear waves. J. Fluids Struct. 13: 607-630

[20] Turnbull M S, Borthwick A G L and Taylor R E 2003 Numerical wave tank based on a $\sigma$-transformed finite element inviscid flow solver. Int. J. Numer. Method. Fluids 42: 641-663

[21] Frandsen J B 2004 Sloshing in excited containers. J. Comput. Phys. 196: 53-87

[22] Chen B F and Nokes R 2005 Time-independent finite difference analysis of fully non-linear and viscous fluid sloshing in a rectangular tank. J. Comput. Phys. 209: 47-81

[23] Dai L and Xu L 2006 A numerical scheme for dynamic liquid sloshing in horizontal cylindrical containers. Proc. Inst. Mech. Eng. Part D: J. Automob. Eng. 20: 901-918

[24] Wu C H, Faltinsen O M and Chen B F 2012 Time-independent finite difference and ghost cell method to study sloshing liquid in 2D and 3D tanks with internal structures. Commun. Comput. Phys. 13(3): 780-800

[25] Eswaran M and Reddy G R 2016 Numerical simulation of tuned liquid tank-structure systems through $\sigma$-transformation based fluid-structure coupled solver. Wind Struct. Int. J. 23(5): 421-447

[26] American Concrete Institute Committee 2006 Seismic design of liquid-containing concrete structures. ACI 350.3-06 and commentary 350.3R-06, Farmington Hills, Michigan, USA: American Concrete Institute

[27] Housner G W 1963 Dynamic analysis of fluids in containers subjected to acceleration. In: Nuclear reactors and earthquakes, Report No. TID 7024, Washington, DC, USA: US Atomic Energy Commission

[28] Frandsen J B 2005 Numerical predictions of tuned liquid tank structural systems. J. Fluids Struct. 20: 309-329

[29] Faltinsen O M, Rognebakke O F, Lukovsky I A and Timokha A N 2000 Multidimensional modal analysis of nonlinear sloshing in a rectangular container with finite water depth. $J$. Fluid Mech. 407: 201-234

[30] Stone H L 1968 Iterative solution of implicit approximations of multidimensional partial differential equations. SIAM J. Numer. Anal. 5: 530-558 
[31] Ferziger J H and Peric M 2002 Computational methods for fluid dynamics. 3rd rev. ed., Berlin, Heidelberg, New York: Springer-Verlag

[32] De A K 2014 An implicit non-staggered Cartesian grid method for incompressible viscous flows in complex geometries. Sadhana 39(5): 1071-1094

[33] Van der Vorst 1992 Bi-CGSTAB: a fast and smoothly converging variant of $\mathrm{Bi}-\mathrm{CG}$ for the solution of non- symmetric linear systems. SIAM J. Sci. Stat. Comput. 13(2): 631-644

[34] Shih M H and Sung W P 2013 Development of semi-active hydraulic damper as active interaction control device to withstand external excitation. Sadhana 39(1), 123-138

[35] Sriram V, Sannasiraj S A and Sundar S 2006 Numerical simulation of 2D sloshing waves due to horizontal and vertical random excitation. Appl. Ocean Res. 28: 19-32 